\title{
Habitable planets around the star Gliese 581?
}

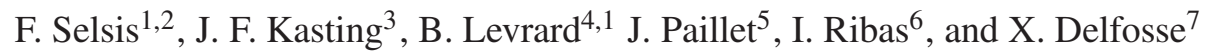 \\ ${ }^{1}$ CRAL: Centre de Recherche Astrophysique de Lyon (CNRS; Université de Lyon; École Normale Supérieure de Lyon), \\ 46 allée d'Italie, 69007 Lyon, France \\ e-mail: franck.selsis@ens-lyon.fr \\ 2 LAB: Laboratoire d'Astrophysique de Bordeaux (CNRS; Université Bordeaux I), BP 89, 33270 Floirac, France \\ 3 Dept. of Geosciences, The Pennsylvania State University, University Park, Pennsylvania 16802, USA \\ e-mail: kasting@geosc.psu.edu \\ 4 IMCCE: Institut de Mécanique Céleste et de Calcul des Ephémérides (CNRS; Université Pierre et Marie Curie - Paris VI), \\ 77 avenue Denfert-Rochereau, 75014 Paris, France \\ e-mail: Benjamin.Levrard@imcce.fr \\ 5 ESA/ESTEC SCI-SA, Keplerlaan 1, PO Box 299, 2200AG Noordwijk, The Netherlands \\ e-mail: jpaillet@rssd.esa.int \\ ${ }^{6}$ Institut de Ciències de l'Espai (CSIC-IEEC), Campus UAB, 08193 Bellaterra, Spain \\ e-mail: iribas@ieec.uab.es \\ 7 LAOG: Laboratoire d'AstrOphysique de Grenoble (CNRS; Université J. Fourier - Grenoble I), BP 53X, 38041 Grenoble Cedex, \\ France \\ e-mail: delfosse@obs.ujf-grenoble.fr
}

Received 15 June 2007 / Accepted 26 October 2007

\section{ABSTRACT}

\begin{abstract}
Context. Thanks to remarkable progress, radial velocity surveys are now able to detect terrestrial planets at habitable distance from low-mass stars. Recently, two planets with minimum masses below $10 M_{\oplus}$ have been reported in a triple system around the M-type star Gliese 581. These planets are found at orbital distances comparable to the location of the boundaries of the habitable zone of their star.

Aims. In this study, we assess the habitability of planets Gl 581c and Gl 581d (assuming that their actual masses are close to their minimum masses) by estimating the locations of the habitable-zone boundaries of the star and discussing the uncertainties affecting their determination. An additional purpose of this paper is to provide simplified formulae for estimating the edges of the habitable zone. These may be used to evaluate the astrobiological potential of terrestrial exoplanets that will hopefully be discovered in the near future.

Methods. Using results from radiative-convective atmospheric models and constraints from the evolution of Venus and Mars, we derive theoretical and empirical habitable distances for stars of $\mathrm{F}, \mathrm{G}, \mathrm{K}$, and $\mathrm{M}$ spectral types.

Results. Planets Gl 581c and Gl 581d are near to, but outside, what can be considered as the conservative habitable zone. Planet "c" receives 30\% more energy from its star than Venus from the Sun, with an increased radiative forcing caused by the spectral energy distribution of Gl 581. This planet is thus unlikely to host liquid water, although its habitability cannot be positively ruled out by theoretical models due to uncertainties affecting cloud properties and cloud cover. Highly reflective clouds covering at least $75 \%$ of the day side of the planet could indeed prevent the water reservoir from being entirely vaporized. Irradiation conditions of planet " $\mathrm{d}$ " are comparable to those of early Mars, which is known to have hosted surface liquid water. Thanks to the greenhouse effect of $\mathrm{CO}_{2}$-ice clouds, also invoked to explain the early Martian climate, planet "d" might be a better candidate for the first exoplanet known to be potentially habitable. A mixture of several greenhouse gases could also maintain habitable conditions on this planet, although the geochemical processes that could stabilize such a super-greenhouse atmosphere are still unknown.
\end{abstract}

Key words. astrobiology - atmospheric effects - stars: planetary systems

\section{Introduction}

The M-type star Gl 581 hosts at least 3 planets, which were detected using radial velocity measurements by Bonfils et al. (2005) (planet "b") and Udry et al. (2007) (planets "c" and "d"). The properties of this star and its planets are given in Table 1. Before this discovery, only two exoplanets were known to have a minimum mass below $10 M_{\oplus}$, which is usually considered as a boundary between terrestrial and giant planets, the latter having a significant fraction of their mass in an $\mathrm{H}_{2}-\mathrm{He}$ envelope. The first one was GJ 876d, a very hot planet $(P \leq 2$ days) with a minimum mass of $5.9 M_{\oplus}$ (Rivera et al. 2005). The other one is OGLE-05-390L b, found to be a $\sim 5.5 M_{\oplus}$ cold planet at
2.1 AU from its low-mass parent star thanks to a microlensing event (Beaulieu et al. 2006; Ehrenreich et al. 2006). Neither of these two planets is considered as habitable, even with very loose habitability criteria. In the case of Gl 581, and as already mentioned by Udry et al. (2007), the locations of planet "c" and "d" must be fairly close to the inner and outer edges, respectively, of the habitable zone (HZ). In this paper, we investigate the atmospheric properties that would be required to make the habitability of these planets possible.

Because of its equilibrium temperature of $\sim 300 \mathrm{~K}$ when calculated with an albedo of 0.5 , it has been claimed that the second planet of this system, Gl 581c, is potentially habitable (Udry et al. 2007), with climatic conditions possibly similar to 
Table 1. Properties of the star Gl 581 and its 3 detected planets, from Udry et al. (2007).

\begin{tabular}{lllll}
\hline \hline Star & $T_{\text {eff }}(\mathrm{K})$ & $M / M_{\odot}$ & $R / R_{\odot}$ & $L / L_{\odot}$ \\
\hline Gl 581 & 3200 & 0.31 & 0.38 & 0.0135 \\
\hline Planets & $a(\mathrm{AU})$ & $M_{\min } / M_{\oplus}$ & $R_{\min } / R_{\oplus}$ & Stellar flux \\
& & ${ }_{*}^{*}$ & $S / S_{0}{ }^{* * *}$ \\
\hline $\mathrm{b}$ & 0.041 & 15.6 & $2.2-2.6$ & 8.1 \\
$\mathrm{c}$ & 0.073 & 5.06 & $1.6-2.0$ & 2.55 \\
$\mathrm{~d}$ & 0.253 & 8.3 & $1.8-2.2$ & 0.21 \\
\hline
\end{tabular}

The potential habitability of planets "c" and "d", highlighted in grey, is discussed in this paper.

${ }^{*} M_{\min }=M \sin i$, where $i$ is the orbital inclination.

** Radius for a rocky and ocean planet, respectively (Sotin et al. 2007; Valencia et al. 2007b).

${ }^{* * *} S_{0}$ is the solar flux at $1 \mathrm{AU}: 1360 \mathrm{~W} \mathrm{~m}^{-2}$.

those prevailing on Earth. After a brief discussion about the relationship between the equilibrium temperature and habitability, we summarize in this paper what are usually considered as the boundaries of the circumstellar $\mathrm{HZ}$ and the uncertainties on their precise location. In Sect. 2.4 we provide parameterizations to determine such limits as a function of the stellar luminosity and effective temperature. These can be used to evaluate the potential habitability of the terrestrial exoplanets that should soon be discovered. We then discuss the specific case of the system around Gl 581.

\section{Habitable planets and the habitable zone}

The HZ is the circumstellar region inside which a terrestrial planet can hold permanent liquid water on its surface. A terrestrial planet that is found beyond the $\mathrm{HZ}$ of its star could still harbor life in its subsurface; but being unable to use starlight as a source of energy, such endolithic biosphere would not be likely to modify its planetary environment in an observable way (Rosing 2005). In the Solar System, in situ searches for biological activity in the subsurface of, for instance, Mars or Jupiter's satellite Europa could in principle be carried out. But with exoplanets presumably out of reach for in situ exploration, signs of life will have to be searched via signatures of photosynthetic processes in the spectra of planets found in the $\mathrm{HZ}$ of their stars. This is the purpose of future space observatories such as Darwin (Volonte et al. 2000; Kaltenegger \& Fridlund 2005), TPF-C (Levine et al. 2006) and TPF-I (Beichman et al. 1999). For exoplanets, "habitable" thus implies surface habitability.

A planet found in the HZ is not necessary habitable. The maintenance of habitable conditions on a planet requires various geophysical and geochemical conditions. Only some of them, those that have a direct influence on the atmospheric properties, are addressed in the present paper (see for instance Scalo et al. 2007; Zahnle et al. 2007; Kasting \& Catling 2003; Lunine 1999; Gaidos \& Selsis 2007, for a comprehensive view of habitability). Many factors may prevent (surface) habitability. To give several examples: the planet may lack water, the rate of large impacts may be too high, the minimum set of ingredients necessary for the emergence of life (so far unknown) may have not been there, gravity may be too weak (as on Mars) to retain a dense atmosphere against escape processes and to keep an active geology replenishing the atmosphere of $\mathrm{CO}_{2}$, or the planet could have accreted a massive $\mathrm{H}_{2}-\mathrm{He}$ envelope that would prevent water from being liquid by keeping the surface pressure too high. To avoid the two last scenarios, the planetary mass should be in the approximate range of $0.5-10 M_{\oplus}$, although this is more of an educated guess than a reliable quantitative estimate.

Being at the right distance from its star is thus only one of the necessary conditions required for a planet to be habitable. In the current absence of observational constraints, we choose to assess the habitable potential of the planets with as few hypotheses as possible on their physical and chemical nature. We therefore assume that the planet satisfies only two conditions. Although these two conditions are very simple, they may derive from complex geophysical properties. Future observations will hopefully tell us whether such properties are frequent or rare on terrestrial exoplanets. These conditions are:

i) The amount of superficial water must be large enough so that the surface can host liquid water for any temperature between the temperature at the triple point of water, $273 \mathrm{~K}$, and the critical temperature of water, $T_{\mathrm{c}}=647 \mathrm{~K}$. This condition implies that the water reservoir produces a surface pressure higher than 220 bars when fully vaporized. With an Earth gravity, this corresponds to a $2.2 \mathrm{~km}$ layer of water, slightly lower than the mean depth of Earth oceans of $2.7 \mathrm{~km}$. For a gravity twice that of Earth, this pressure corresponds to half this depth. Planets with less water may still be habitable, but their HZs may be somewhat narrower than we calculate here because liquid water would disappear at a lower surface temperature.

ii) Atmospheric $\mathrm{CO}_{2}$ must accumulate in a planet's atmosphere whenever the mean surface temperature falls below $273 \mathrm{~K}$, the freezing point of water. This is a consequence of the carbonate-silicate cycle, which stabilizes the long-term surface temperature and the amount of $\mathrm{CO}_{2}$ in the atmosphere of the Earth (Walker et al. 1981). Such an assumption implies that the planet is geologically active and continuously outgassing $\mathrm{CO}_{2}$. It also implies that carbonates form in the presence of surface liquid water, which may require continental weathering. With no atmospheric $\mathrm{CO}_{2}$, or with a fixed $\mathrm{CO}_{2}$ level as in Hart (1979), the HZ could be $\sim 10$ times narrower than is currently assumed. In the absence of $\mathrm{CO}_{2}$ (or a greenhouse gas other than $\mathrm{H}_{2} \mathrm{O}$ ), the present Earth would be frozen.

The atmosphere of a habitable planet meeting these conditions should behave as illustrated in Fig. 1.

\subsection{The equilibrium temperature}

The equilibrium temperature of a planet is given by

$T_{\text {eq }}=\left(\frac{S(1-A)}{f \sigma}\right)^{\frac{1}{4}}$

where $A$ is the Bond albedo (which is the fraction of power at all wavelengths scattered back out into space - Earth's value is 0.29), $S$ is the stellar energy flux, $\sigma$ is the Stefan-Boltzmann constant, and $f$ is a redistribution factor. If all the incident energy is uniformly distributed on the planetary sphere, then $f=4$. If the energy is uniformly distributed over the starlit hemisphere alone, then $f=2$. And if there is no redistribution, the local equilibrium temperature can be calculated with $f=1 / \cos \theta$ where $\theta$ is the zenith angle. The latter case, for instance, yields good results for the surface temperature on the sunlit hemispheres of airless bodies with known albedo such as the Moon and Mercury.

It is important to discuss the meaning of $T_{\mathrm{eq}}$ and the manner in which it can be used to assess habitability. The planet Gl 581c 


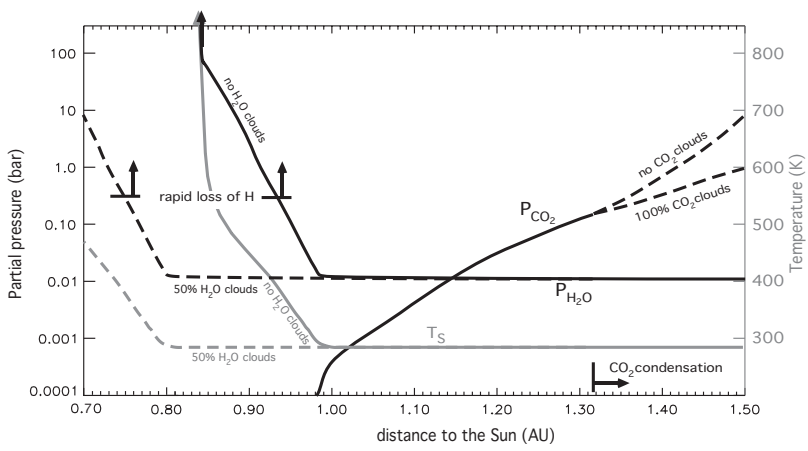

Fig. 1. The $\mathrm{CO}_{2}$ and $\mathrm{H}_{2} \mathrm{O}$ pressure and the mean surface temperature of a habitable planet as a function of the orbital distance. The diagram gives $P_{\mathrm{CO}_{2}}$ and $\mathrm{P}_{\mathrm{H}_{2} \mathrm{O}}$ (left $y$-axis) and $T_{S}$ (grey, right $y$-axis) of an Earthlike planet across the inner part of the $\mathrm{HZ}$ around the present Sun. At orbital distances larger than $\sim 0.98 \mathrm{AU}, T_{S}$ is assumed to be fixed at $288 \mathrm{~K}$ (its current value on Earth) by the carbonate-silicate cycle, which is obviously an idealized picture. Beyond $1.3 \mathrm{AU}, \mathrm{CO}_{2}$ condenses in the atmosphere and the required level of $\mathrm{CO}_{2}$ depends on the coverage by $\mathrm{CO}_{2}$-ice clouds. The pressure of $\mathrm{H}_{2} \mathrm{O}$ and $T_{\mathrm{s}}$ in the inner $\mathrm{HZ}$ is calculated for a cloud-free atmosphere and $50 \%$ cloud cover and assuming a reservoir of water that contains (as on Earth) more than the equivalent of 220 bars of $\mathrm{H}_{2} \mathrm{O}$.

has been widely presented as potentially habitable because one finds $T_{\text {eq }} \sim 320 \mathrm{~K}$ when calculated using the albedo of the Earth. This conclusion is however too simplistic for the following two reasons:

i) For a planet with a dense atmosphere (an inherent property of a habitable planet), $T_{\text {eq }}$ does not indicate any physical temperatures at the surface or in the atmosphere. With albedos of $0.75,0.29$, and 0.22 , respectively, and assuming $f=4$, Venus, Earth, and Mars have equilibrium temperatures of $231 \mathrm{~K}, 255 \mathrm{~K}$, and $213 \mathrm{~K}$, while their mean surface temperatures are $737 \mathrm{~K}, 288 \mathrm{~K}$ and $218 \mathrm{~K}$. The two quantities only match, approximately, in the case of Mars, whose tenuous atmosphere produces a greenhouse warming of only $\sim 5 \mathrm{~K}$.

ii) It can be demonstrated that a necessary (but not sufficient) condition for habitability is that $T_{\text {eq }}$ must be lower than about $270 \mathrm{~K}$. If the surface temperature remains below the critical temperature of water $\left(T_{\mathrm{c}}=647 \mathrm{~K}\right)$, the thermal emission of a habitable planet cannot exceed the runaway greenhouse threshold, $\sim 300 \mathrm{~W} \mathrm{~m}^{-2}$ (see Sect. 2.2.1), equivalent to the irradiance of a black-body at $270 \mathrm{~K}$. Therefore, if a planet has an atmosphere and an equilibrium temperature above $270 \mathrm{~K}$, two situations may arise. First, $T_{\mathrm{s}}$ may remain below $T_{\mathrm{c}}$, but there would be no liquid water at the surface and no or negligible amounts of water vapor in the atmosphere. In a second possible situation, the atmosphere contains considerable amounts of water vapor, but the surface temperature exceeds $1400 \mathrm{~K}$ (see Sect. 2.2.1). This would allow the planet to balance the absorbed stellar energy by radiating at visible and radio wavelengths through an atmosphere that is optically thick in the infrared (IR). Both cases would render the planet uninhabitable.

For planet Gl 581c to be habitable (i.e., $T_{\text {eq }}<270 \mathrm{~K}$ ), its albedo would have to be higher than 0.65 . Since planet Venus has an albedo of 0.75 , this situation may not appear unrealistic. However, as we will see in the next sections, the Bond albedo is not a quantity given by the planetary characteristics alone, but the spectral energy distribution of the star also needs to be taken into account.

\subsection{The inner edge of the $H Z$}

Let us consider a planet with a large water reservoir covering its entire surface, but no other greenhouse volatiles. As a first step, we assume that its host star is a Sun-like star and that the planet has the same gravity as the Earth. For a given orbital distance, a fraction of the water reservoir is in the form of vapor. The surface temperature $T_{\mathrm{s}}$ imposes the surface vapor pressure $P_{\mathrm{w}}$. If $P_{\mathrm{w}}$ is high enough, the water vapor, in turn, affects $T_{\mathrm{s}}$ by blocking the outgoing IR radiation, by reducing the atmospheric lapse rate and by modifying the planetary albedo. To account for this coupling, the atmospheric structure has to be computed selfconsistently for a given irradiation. This was done previously by using a 1D radiative-convective model (Kasting 1988). All the orbital distances and stellar fluxes in the following subsections are given relative both to the present Sun and to the present solar flux at Earth orbit $\left(S_{0}=1360 \mathrm{~W} \mathrm{~m}^{-2}\right)$. We will see further how these values can be scaled to other stellar luminosities and effective temperatures. Values given in Sects. 2.2.1-2.2.3 were obtained by Kasting (1988) with a cloud-free radiative convective scheme. Section 2.2.5 discusses the likely effects of clouds.

\subsubsection{The runaway greenhouse limit}

For orbital distances smaller than $1 \mathrm{AU}$ (and for the present solar luminosity), $T_{\mathrm{S}}$ is extremely sensitive to the orbital distance, increasing from less than $273 \mathrm{~K}$ at $1 \mathrm{AU}$ (in the absence of $\mathrm{CO}_{2}$ ) to about $373 \mathrm{~K}\left(P_{\mathrm{w}}=1\right.$ bar) at $0.95 \mathrm{AU}$ (see Fig. 1). This sharp increase in $T_{\mathrm{s}}$ is mainly caused by the increase in the IR opacity and the decrease in the albedo caused by absorption of solar near-infrared (NIR) radiation by water vapor. For even smaller orbital distances, because of the relation between temperature, vapor pressure, and IR opacity, the outgoing IR flux becomes nearly independent of the surface temperature and tends asymptotically towards its upper limit of about $300 \mathrm{~W} \mathrm{~m}^{-2}$, known as the runaway greenhouse threshold (Abe \& Matsui 1988; Kasting 1988; Ishiwatari et al. 2002). At this point, an increase in the irradiation (or a decrease of the orbital distance) does not result in an increase in the outgoing IR flux, but leads instead to a strong increase in $T_{\mathrm{s}}$ and $P_{\mathrm{w}}$. In turn, this produces a slight increase in the albedo and thus in the reflected visible/NIR radiation. The increase in the albedo for $T_{\mathrm{s}}$ above $373 \mathrm{~K}$ and $P_{\mathrm{w}}$ above 1 bar is the consequence of the strong Rayleigh back-scattering occurring in the visible (a spectral domain where water vapor does not absorb significantly) and to the saturation of the water bands absorbing the stellar NIR radiation. This increase in the atmospheric albedo, up to about 0.35 (in the absence of clouds), protects the water reservoir from complete vaporization for orbital distances down to $0.84 \mathrm{AU}\left(1.4 S_{0}\right)$. At $0.84 \mathrm{AU}, T_{\mathrm{s}}$ reaches $T_{\mathrm{c}}=647 \mathrm{~K}$, and the water reservoir becomes a supercritical fluid envelope. A more limited water reservoir could, of course, be fully vaporized at lower irradiation. When this theoretical limit for the irradiation is crossed, there is a dramatic increase in $T_{\mathrm{s}}$ from $>647 \mathrm{~K}$ to $>1400 \mathrm{~K}$, a temperature that potentially melts silicates on the surface. This behavior is a consequence of the runaway greenhouse threshold that limits the mid-IR cooling of the planet. At $T_{\mathrm{s}}>1400 \mathrm{~K}$, the planet can radiate the absorbed stellar energy through the atmosphere at the visible and radio wavelengths, at which the water vapor opacity is negligible.

\subsubsection{The water loss limit}

In a cloud-free radiative-convective scheme, water vapor would become a major atmospheric constituent in an Earth analog 
placed at 0.95 AU from the present Sun. The loss of hydrogen to space would no longer be limited by the diffusion of water vapor from the troposphere to the stratosphere, but by the stellar EUV energy deposited in the upper atmosphere, and would be enhanced by $\sim 4$ orders of magnitude. The hydrogen contained in the whole terrestrial ocean would thus be lost in less than $1 \mathrm{Gyr}$, which would terminate Earth's habitability. For these two reasons, $0.95 \mathrm{AU}$ could be seen as the inner limit of the present solar HZ.

The water loss limit is difficult to extrapolate to other stars and terrestrial exoplanets. Such a limit for present Earth corresponds to a surface temperature of $\sim 340 \mathrm{~K}$. This is because the background atmospheric pressure is 1 bar (mainly $\mathrm{N}_{2}$ and $\mathrm{O}_{2}$ ), and the vapor pressure of $\mathrm{H}_{2} \mathrm{O}$ at $340 \mathrm{~K}$ is 0.2 bar, making $\mathrm{H}_{2} \mathrm{O}$ a $20 \%$ constituent of the atmosphere. At this $\mathrm{H}_{2} \mathrm{O}$ mixing ratio, the loss of $\mathrm{H}$ to space becomes energy-limited. If the background surface pressure is higher, then the water-loss limit will be reached at a higher temperature. For instance, with a background atmospheric pressure of 5 bars, the water loss limit would be reached at about $373 \mathrm{~K}$. In addition, the water content of terrestrial planets is thought to be highly variable (Raymond et al. 2007 ) and could be as high as $50 \%$ in mass for migrating planets initially formed beyond the snow line (Kuchner 2003; Léger et al. 2004; Selsis et al. 2007). For such water-rich planets, only a fraction of the water can be lost within the lifetime of the planet, and atmospheric escape is not a threat to habitability.

Planets in the Gl 581 system are good ocean planet candidates because the architecture of the system and the high mass of the planets are likely to be inherited from type-I migration. These planets can thus be initially composed of a large fraction of water ice. In addition, the lifetime of the water reservoir also depends on the stellar emission in the UV, which photodissociates $\mathrm{H}_{2} \mathrm{O}$, and in the XUV $(0.1-100 \mathrm{~nm})$, which produces thermospheric heating. The star Gl 581 appears quite inactive and should not have strong UV or XUV fluxes presently. Thus, gravitational escape is not likely to play a major role in the system today. However, the high-energy emissions of Gl 581 may have been orders of magnitude stronger in the past. The evolution of these emissions has not been accurately established for M-type stars yet, but they are likely to have had an impact on the planet's atmosphere for a more or less extended period of time. This point is addressed in more detail in Sect. 3.4.

As already mentioned, the $\mathrm{HZ}$ comprises the orbital regions where terrestrial planets can be probed in search of biosignatures. In this context, water loss is an important issue, because the leftover oxygen could possibly produce a dense $\mathrm{O}_{2}$-rich atmosphere. This may be impossible to distinguish from an atmosphere sustained by photosynthesis, unless additional biomarker gases were also detected. Therefore, planets found closer to their parent star than the water-loss limit represent questionable astrobiological targets.

\subsubsection{The thermophilic limit}

Terrestrial organisms have the extraordinary ability to adapt themselves to extreme conditions, including hot environments. For hyperthermophilic prokaryotes, the optimum temperature for growth is above $353 \mathrm{~K}$. Pyrolobus fumarii, an iron-breathing bacterium, has been found to tolerate temperature as high as $394 \mathrm{~K}$, setting the record for the highest temperature known to be compatible with life. This temperature limit is lower $(\sim 333 \mathrm{~K})$ for eukaryotes and photosynthetic prokaryotes. The orbital distance at which $T_{\mathrm{s}}$ cannot be lower than $394 \mathrm{~K}$ could thus be seen as an empirical, anthropocentric, inner edge of the HZ.
Interestingly, this highest temperature tolerated by life (as we know it) is close to the mean surface temperature at which the loss of water becomes considerable, so that this limit and the water-loss limit are located at approximately the same orbital distance of $\sim 0.94 \mathrm{AU}$ for a cloud-free planet.

\subsubsection{The Venus criterion}

Radar maps of the surface of Venus suggest that liquid water has not been present there for at least the last 1 Gyr (Solomon $\&$ Head 1991). The Sun was $~ 8 \%$ dimmer at that time, according to standard solar evolution models (e.g., Baraffe et al. 1998). Thus, the solar flux at Venus' orbit was then equal to what it is today at a distance of $0.72 \mathrm{AU} \times(1 / 0.92)^{0.5} \sim 0.75 \mathrm{AU}$. This provides an empirical indication of the location of the inner edge of the HZ. We do not know if Venus lost its water content after experiencing a runaway greenhouse or because its water reservoir was much smaller than Earth's. The D/H ratio measured in Venusian water vapor traces is $120 \pm 40$ times higher than on Earth (de Bergh et al. 1991). Thus, if the initial D/H ratio of Venusian water was the same as on Earth, there must have been at least $\sim 120$ times more water on early Venus than today. This corresponds to an initial inventory of about $20 \mathrm{~m}$ of precipitable water. Since deuterium is also lost to space, albeit at a slower rate than $\mathrm{H}$, and volcanoes release poorly deuterated hydrogen into Venus' atmosphere, this lower limit can significantly underestimate the initial water content. This reveals that the cold trap limiting the flux of water vapor from the troposphere to the stratosphere did not work on Venus as it worked on Earth. In turn, this implies that 1 Gyr ago or longer the mean surface temperature was high enough to trigger massive water loss $(\sim 340 \mathrm{~K}$ or higher depending of the background atmospheric pressure).

Is it possible to explain the $\mathrm{D} / \mathrm{H}$ ratio of Venus atmosphere if we assume that the current $\mathrm{CO}_{2}$-rich, hot, and mainly dry conditions have always been prevailing at the surface of Venus? The answer is yes, if the deuterium enrichment has been generated by the loss of the water delivered sporadically by impacts. The minimum amount of water lost $\left(\sim 10^{16} \mathrm{~m}^{3}\right.$ in volume, $10^{19} \mathrm{~kg}$ in mass) corresponds to the contents of 25000 Halleysized comets. Venus should not have experienced such an accumulated delivery since the late heavy bombardment (LHB), which occurred 3.95-3.85 Gyr ago. Through numerical simulations, Gomes et al. (2005) found that $10^{19} \mathrm{~kg}$ of cometary material $\left(\sim 5 \times 10^{18} \mathrm{~kg}\right.$ of water) impacted on the Moon during the LHB. When scaling this total impact volume to Venus (by assuming a factor 1-10 increase relative to the Moon) and taking the cometary $\mathrm{D} / \mathrm{H}$ ratio (twice as large as the terrestrial ocean value) into account, we can see that enough water could have been brought to Venus during the LHB. However, if this water was lost soon after the LHB, deuterium has then been escaping during the last $3.85 \mathrm{Gyr}$ and only a small fraction should remain today. This implies a much higher water delivery that is unlikely to be consistent with post-accretion impacts. In summary, although impact-delivered water is unlikely to explain the present $\mathrm{D} / \mathrm{H}$ enrichment of Venus, we must keep in mind that the Venus criterion assumes that the lost water was condensed on the surface at some point during Venus' history, which has not been proved.

\subsubsection{The effect of clouds on the inner boundary of the $\mathrm{HZ}$}

On a habitable planet close to the inner boundary of the HZ, the IR opacity of the atmosphere is fully dominated by water vapor, 
and clouds do not contribute to warming the surface, as some types of clouds (high cirrus clouds) can do on Earth. But, on the other hand, clouds can significantly increase the planetary albedo and thereby reduce the greenhouse warming. In particular, thick clouds forming at high altitude, above the level where the incident radiation is backscattered or absorbed, can result in a very high albedo and can thus move the habitability limits closer to the star. The $1 \mathrm{D}$ radiative convective models that have been used to estimate the climatic response to an increase of stellar flux (Kasting 1988, 1991) can only bracket the quantitative effects of clouds on the planetary radiation budget. Clouds are by nature a $3 \mathrm{D}$ phenomenon that is closely related to atmospheric circulation. By adding a cloud layer in a $1 \mathrm{D}$ model, it is however possible to investigate the effect of a $100 \%$ cloud cover. We can also estimate the value of $T_{\mathrm{s}}$ with a $50 \%$ cloud coverage (or any other percentage) by assuming that clouds do not affect the IR outgoing radiation. This allows us to calculate the total albedo by combining cloud-free and cloudy models and to find the orbital distance at which the absorbed energy matches the IR outgoing radiation. This approximation is acceptable for large enough water vapor columns, which are found for $T_{\mathrm{s}}$ roughly above $373 \mathrm{~K}$, and thus for calculations near the inner edge. The $T_{\mathrm{s}}$ and $P_{\mathrm{w}}$ curves for the $50 \%$ cloud coverage case in Fig. 1 are thus reliable only in the innermost part of the HZ (for $T_{\mathrm{s}}>373 \mathrm{~K}$ ).

A cloud layer located between the 0.1 and 1 bar levels (just above the surface in the case of present Earth, but at an altitude of $\sim 150 \mathrm{~km}$ for a hotter planet with more than 100 bar of $\mathrm{H}_{2} \mathrm{O}$ ) produces the maximum increase in the albedo. For a planet orbiting our present Sun, these clouds can produce an albedo as high as 0.8 and 0.6, respectively, when covering $100 \%$ and $50 \%$ of the day side. This would move the runaway greenhouse limit to $0.46 \mathrm{AU}(100 \%)$ and $0.68 \mathrm{AU}(50 \%)$ from the Sun. Interestingly, the water-loss limit $\left(T_{\mathrm{s}}=373 \mathrm{~K}\right)$ with $50 \%$ cloudiness matches the empirical Venus limit (0.72 AU). Such high-altitude and thick clouds form mainly by condensation in updrafts, and hence are unlikely to cover a planet's entire hemisphere. Joshi (2003) simulated the 3D climate of a synchronously rotating planet (which is expected for planets in circular orbits within the HZ of M stars, see Sect. 3.5), with various ocean/continent ratios. The author found that the high-altitude clouds were mainly located on the dark side of the planet and that the cloud cover on the day side was below 50\%. This simulation was calculated for a thin Earth-like atmosphere and should not be used to constrain the cloudiness of a thick $\mathrm{H}_{2} \mathrm{O}$-rich atmosphere close to the inner edge of the HZ. It is, however, a good illustration of the simulations that should be developed in the future to address the meteorology of exoplanets.

\subsection{The outer edge of the $H Z$}

When the carbonate-silicate cycle is at work, the level of $\mathrm{CO}_{2}$ should be stabilized at a value sustaining a mean surface temperature $T_{\mathrm{s}}$ somewhere above $273 \mathrm{~K}$. It has to be more than a few degrees above $273 \mathrm{~K}$ because of the runaway ice-albedo feedback that would trigger a global glacial event if a relatively large fraction of the oceans is frozen. The precise stabilizing temperature should depend on the planet's land/ocean distribution, internal heat flow, surface carbon inventory, and other factors that are difficult to constrain and that may vary from planet to planet.

Note that models computing the fluxes between the different $\mathrm{CO}_{2}$ reservoirs (atmosphere, ocean, biosphere, crust, mantle) and the consequent $\mathrm{CO}_{2}$ atmospheric level on an Earthlike planet do exist (Franck et al. 2002; Sleep \& Zahnle 2001).
Unfortunately, such models typically depend on planetary parameters (bulk composition, volatile content, radiogenic elements abundances, structure, formation history, internal energy, heat flux ,...) that are impossible to estimate before a planet has actually been discovered and deeply characterized.

Considering the limited understanding of the carbon cycle on Earth, and the expected diversity of exoplanets, we make the very simple assumption that the $\mathrm{CO}_{2}$ level is potentially maintained at a habitable level. If it is not, then the planet is obviously not habitable, which recalls that being inside the habitable zone is a necessary but not sufficient condition for habitability.

The relationship between the orbital distance and the required level of $\mathrm{CO}_{2}$ is given in Fig. 1, where the stabilization temperature is arbitrarily fixed to $288 \mathrm{~K}$, the current value of $T_{\mathrm{S}}$ on Earth. At $1 \mathrm{AU}$ from the present Sun, a $\mathrm{CO}_{2}$ abundance of $3 \times 10^{-4}$ bar is sufficient to keep $T_{\mathrm{s}}$ of the Earth at $288 \mathrm{~K}$. Four billion years ago, the solar luminosity was about $70 \%$ of its present value, and 1000 times more $\mathrm{CO}_{2}$ should have been required to maintain the same $T_{\mathrm{s}}$. If the Earth was placed at 1.2 $\mathrm{AU}$ from the present Sun, the $\mathrm{CO}_{2}$ level would stabilize at approximately this same high value. The farthest orbital distance at which the Earth could be placed without freezing permanently represents the outer boundary of the HZ. This outer edge has not been accurately determined yet because of the complex effects of clouds resulting from $\mathrm{CO}_{2}$ condensation.

If we consider a cloud-free $\mathrm{CO}_{2}$ atmosphere (with a water pressure fixed by $T_{\mathrm{s}}$ ), the outer edge should lie at $1.67 \mathrm{AU}$ for the present Sun, where a planet would have a $\mathrm{CO}_{2}$ pressure of about 8 bar (Kasting 1991). For $\mathrm{CO}_{2}$ pressures above 6 bar, the cooling caused by the albedo exceeds the warming caused by the IR opacity of the $\mathrm{CO}_{2}$ column. However, this $1.67 \mathrm{AU}$ limit does not take into account the effect of $\mathrm{CO}_{2}$ clouds, which should form a significant cover at orbital distances larger than 1.3 AU.

\subsubsection{The $\mathrm{CO}_{2}$-cloud limit}

The optical properties of $\mathrm{CO}_{2}$-ice clouds differ significantly from those made of water droplets or $\mathrm{H}_{2} \mathrm{O}$-ice particles. Carbon dioxide clouds are more transparent in the visible range, but they efficiently scatter thermal radiation around $10 \mu \mathrm{m}$. Forget \& Pierrehumbert (1997) showed that the warming effect caused by the backscattering of the IR surface emission exceeds the cooling effect caused by the increase of albedo. As a consequence, $\mathrm{CO}_{2}$ condensation increases the greenhouse warming compared to a purely gaseous $\mathrm{CO}_{2}$ atmosphere. Depending on the fractional cloud cover, the theoretical outer edge of the HZ should be found between the 1.67 AU cloud-free limit and a 100\% cloud cover at 2.4 AU (Forget \& Pierrehumbert 1997; Mischna et al. 2000).

\subsubsection{The early Mars criterion}

Numerous geological and geochemical features indicate that liquid water was present on the surface of Mars as early as $4 \mathrm{Gyr}$ ago (Pollack et al. 1987; Bibring et al. 2006), when the luminosity of the Sun was $28 \%$ lower. The solar flux at Mars' orbit was then equal to what it is today at an orbital distance of $1.5 \mathrm{AU} \times$ $(1 / 0.72)^{0.5} \sim 1.77$ AU. Whatever the cause of the greenhouse warming on early Mars, this fact suggests empirically that the outer edge is located beyond this distance. A likely explanation for the early habitability of Mars is the climatic effect of $\mathrm{CO}_{2}$ clouds, perhaps combined with additional warming by reduced greenhouse gases. Most of the geological features associated 

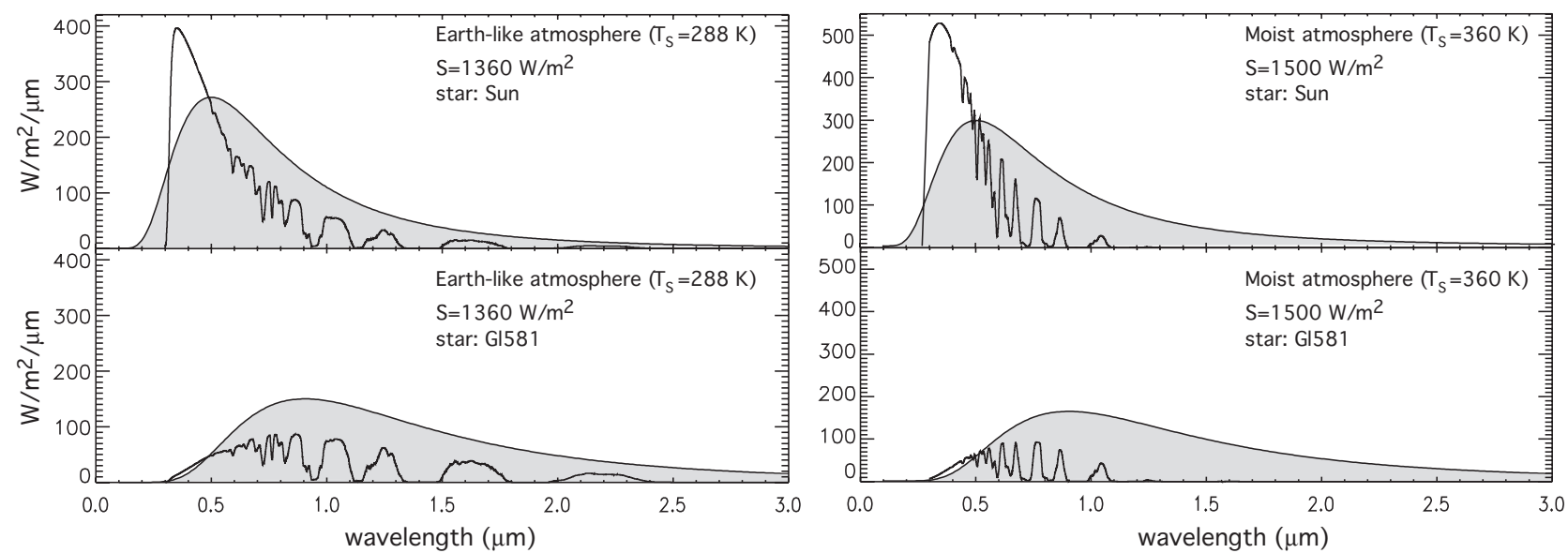

Fig. 2. Plots showing the effect of the stellar effective temperature on the albedo. The graphs represent the reflected irradiance at the substellar point of a planet subject to the irradiation from a Sun-like star with $T_{\text {eff }}=5800 \mathrm{~K}($ top $)$ and from an M-type star with $T_{\text {eff }}=3200 \mathrm{~K}$, similar to Gl 581 (bottom). In this illustration, both stars are assumed to radiate as black bodies. The graphs give the spectral irradiance of a planet receiving an integrated stellar flux of $1360 \mathrm{~W} \mathrm{~m}^{-2}$ (left) and $1500 \mathrm{~W} \mathrm{~m}^{-2}$ (right). The shaded curves with black-body shape give the irradiance of an airless planet with a fixed surface albedo of 0.15 . The non-shaded spectra show the irradiance computed with Phoenix (Paillet 2006) when a cloud-free atmosphere is added. The current Earth atmosphere is shown on the left and a $\mathrm{N}_{2}-\mathrm{H}_{2} \mathrm{O}$ atmosphere with $T_{\mathrm{s}}=360 \mathrm{~K}, P_{\mathrm{S}}=1.3$ bar and $P_{\mathrm{w}}=0.5$ bar on the right. This illustrates the increase in the reflected energy with the stellar effective temperature.

with running water can indeed be explained by a 3D model of the early Martian climate, including clouds, with a $\sim 1$ bar $\mathrm{CO}_{2}$ atmosphere (Forget 2007). Therefore, the outer boundary of the solar HZ must be located somewhere between 1.77 and 2.4 AU.

\subsubsection{A greenhouse-cocktail limit?}

If a planet's atmosphere contains other greenhouse gases in addition to $\mathrm{H}_{2} \mathrm{O}$ and $\mathrm{CO}_{2}$, surface conditions could remain habitable at larger orbital distances than the outer limit defined by the warming of $\mathrm{CO}_{2}$ clouds. Reduced gases, such as methane $\left(\mathrm{CH}_{4}\right)$ or ammonia $\left(\mathrm{NH}_{3}\right)$, could for instance increase the greenhouse effect (Sagan \& Chyba 1997). Methane could have been an important greenhouse gas on early Earth when oxygen was only a trace constituent of the atmosphere. At that time, the photochemical lifetime of atmospheric $\mathrm{CH}_{4}$ was longer and a release of this gas at its present rate would have resulted in an atmospheric level 100-1000 times higher than today. Such a high level of $\mathrm{CH}_{4}$ produces a significant greenhouse warming by absorbing the thermal IR in the $7.4 \mu \mathrm{m}$ band. The efficiency of $\mathrm{CH}_{4}$ as a greenhouse gas has, however, been notably revised downwards by Haqq-Misra et al. (2007) compared to earlier estimates by Pavlov et al. (2000). The IR absorption of $\mathrm{CH}_{4}$ is observable in a global low-resolution mid-IR spectrum, as could be measured by Darwin or TPF-I (Kaltenegger et al. 2007). In fact, a spectrum of a planet's IR emission can potentially reveal any atmospheric species working as a major greenhouse gas.

Since $\mathrm{CO}_{2}$ is not the only greenhouse gas able to maintain habitable conditions at low stellar irradiation, one could use atmosphere models to find what the optimum composition is to produce a mean surface temperature above $273 \mathrm{~K}$ at the largest possible orbital distance. This could be done by adding species transparent to the stellar irradiation but whose combined absorption covers the whole mid-IR. The combined effect of two or more species has to be studied in a self-consistent way. For instance, stratospheric warming due to the NIR absorption of $\mathrm{CH}_{4}$ could induce more cooling than warming by preventing the formation of $\mathrm{CO}_{2}$ clouds. Doing the proper modeling, we may find that a planet containing a cocktail of, say, $\mathrm{CO}_{2}, \mathrm{CH}_{4}, \mathrm{~N}_{2} \mathrm{O}, \mathrm{NH}_{3}$, and CFCs, may extend surface habitability as far as 3 AU from the Sun (or 0.35 AU from Gl 581, see Sect. 3) The problem with this approach is to justify how such a composition could be sustained, taking into account the atmospheric sources and sinks, over geological periods. Life itself could be a possible answer. A planet could be made habitable on purpose by an intelligent civilization able to engineer the atmosphere. "Terraforming" of Mars has for instance been considered (McKay et al. 1991). The greenhouse cocktail could also be maintained in a "Gaia like" homeostasis by some ecosystems that have initially originated in the planet subsurface. We will leave this tantalizing problem to further studies and will consider for the moment that $2.4 \mathrm{AU}$ is the outermost edge of the HZ for a Sun-like star.

\subsection{Limits of the $H Z$ for non solar-type stars}

We have seen that the planetary albedo plays a crucial role in the definition of the HZ. When the absorbed stellar flux per unit surface $S \times(1-A) / 4$ becomes higher than $300 \mathrm{~W} \mathrm{~m}^{-2}$, a runaway greenhouse makes the planet uninhabitable. The albedo of the planetary surface is not important when determining the inner and outer boundaries of the HZ because the albedo of a habitable planet close to these edges would be fully determined by its atmospheric composition (including clouds) and the spectral distribution of the stellar radiation. The Sun emits a large fraction of its energy in the visible, a wavelength domain where the atmosphere of a habitable planet is highly reflective, because of the dependence of Rayleigh backscattering as $\lambda^{-4}$ and because of the lack of strong $\mathrm{H}_{2} \mathrm{O}$ absorption bands. The emission of stars with a low effective temperature peaks in the NIR (around $0.9 \mu \mathrm{m}$ for Gl 581). In the NIR, the contribution of Rayleigh backscattering to the albedo becomes negligible and the strong absorption bands of $\mathrm{H}_{2} \mathrm{O}$ (plus $\mathrm{CO}_{2}$ and possibly $\mathrm{CH}_{4}$ in the outer $\mathrm{HZ}$ ) cause additional absorption of stellar radiation. This effect is illustrated in Fig. 2.

Because of the relation between the albedo and the effective temperature of the star, the limits of the HZ cannot be simply scaled to the stellar luminosity. For stellar effective temperatures between 3700 and $7200 \mathrm{~K}$, Kasting et al. (1993) calculated the albedo of a planet near both edges of the $\mathrm{HZ}$ that has either a dense $\mathrm{H}_{2} \mathrm{O}$ or a dense $\mathrm{CO}_{2}$ atmosphere. Around an M-type star 
Table 2. Boundaries of the present Solar HZ.

\begin{tabular}{lllll}
\hline \hline & Venus & Clouds & Clouds & Clouds \\
& crit. & $0 \%$ & $50 \%$ & $100 \%$ \\
\hline$l_{\text {in॰ }}(\mathrm{AU})$ & 0.72 & $0.84-0.95$ & $0.68-0.76$ & $0.46-0.51$ \\
\hline & Mars & Clouds & Clouds & Clouds \\
& crit. & $0 \%$ & $50 \%$ & $100 \%$ \\
\hline$l_{\text {out } \odot}(\mathrm{AU})$ & 1.77 & 1.67 & 1.95 & 2.4 \\
\hline & & & &
\end{tabular}

with $T_{\text {eff }}=3700 \mathrm{~K}$, for instance, accounting for this effect represents a $40 \%$ difference in the stellar flux (15\% in orbital distance) for a cloud-free $\mathrm{H}_{2} \mathrm{O}$-rich atmosphere close to the inner edge. The scaling factor for a planet with a thin atmosphere, like the modern Earth, is smaller - about $10 \%$ in terms of stellar flux, or 5\% in semi-major axis (Segura et al. 2005). From the various cases studied by Kasting et al. (1993), the limit $l_{\text {in }}$ and $l_{\text {out }}$ of the solar HZ can be extrapolated to any star with luminosity $L$ and an effective temperature between $3700 \mathrm{~K}$ and $7200 \mathrm{~K}$ by using the following relationships:

$$
\begin{gathered}
l_{\text {in }}=\left(l_{\text {in } \odot}-a_{\text {in }} T_{\star}-b_{\text {in }} T_{\star}^{2}\right)\left(\frac{L}{L_{\odot}}\right)^{\frac{1}{2}} \\
l_{\text {out }}=\left(l_{\text {out } \odot}-a_{\text {out }} T_{\star}-b_{\text {out }} T_{\star}^{2}\right)\left(\frac{L}{L_{\odot}}\right)^{\frac{1}{2}}
\end{gathered}
$$

with $a_{\text {in }}=2.7619 \times 10^{-5}, b_{\text {in }}=3.8095 \times 10^{-9}, a_{\text {out }}=1.3786 \times$ $10^{-4}, b_{\text {out }}=1.4286 \times 10^{-9}$, and $T_{\star}=T_{\text {eff }}-5700$. Here, $l_{\text {in }}$ and $l_{\text {out }}$ are in $\mathrm{AU}$, and $T_{\text {eff }}$ in $\mathrm{K}$.

As discussed above, the values of $l_{\text {in॰ } \odot}$ and $l_{\text {out } \odot}$ depend on the criteria chosen to define the limit of habitability. Table 2 gives the limits of the present solar HZ based on the "early Mars" and "recent Venus" criteria, and the radiative-convective models with a cloudiness of 0,50 , and $100 \%$. For each cloud coverage, two $l_{\text {in } \odot ~}$ values are given: the runaway greenhouse limit, and the $T_{\mathrm{s}}=$ 373 K limit.

Note that the effect of the spectral type on the albedo, included in Eqs. (2) and (3) as a quadratic function of $\left(T_{\text {eff }}-5700\right)$, was estimated only for a cloud-free atmosphere. Since the reflectivity of clouds is less sensitive to wavelength, this quadratic term may not be valid to scale the boundaries of the $\mathrm{HZ}$ for planets covered by clouds. The actual boundary locations can be bracketed by using the simple luminosity scaling and Eqs. (2) or (3) that include the $T_{\text {eff }}$ sensitivity.

\subsubsection{The continuously habitable zone}

The luminosity of a star, and thus the boundaries of its HZ, change during its lifetime. Therefore, another criterion for planetary habitability could be the time spent at a habitable distance. For any star of a given spectral type, it is possible to define the limits of the region that remains within the $\mathrm{HZ}$ for a time period longer than a selected timespan. Figure 3 shows the boundaries of the continuously $\mathrm{HZ}(\mathrm{CHZ})$ as a function of the stellar mass, computed with Eqs. (2) and (3) and with an evolutionary model for stars of solar metallicity (Baraffe et al. 1998). Since Eqs. (2) and (3) are not valid for stellar $T_{\text {eff }}$ below $3700 \mathrm{~K}$, the effect on the albedo was calculated by assuming $T_{\text {eff }}=3700 \mathrm{~K}$ for temperatures below this value.

\section{The cases of Gliese 581c and Gliese 581d}

The star Gl 581 has an inferred effective temperature of $3200 \mathrm{~K}$ and a luminosity of $0.013 L_{\odot}$ (Udry et al. 2007). Equations (2)

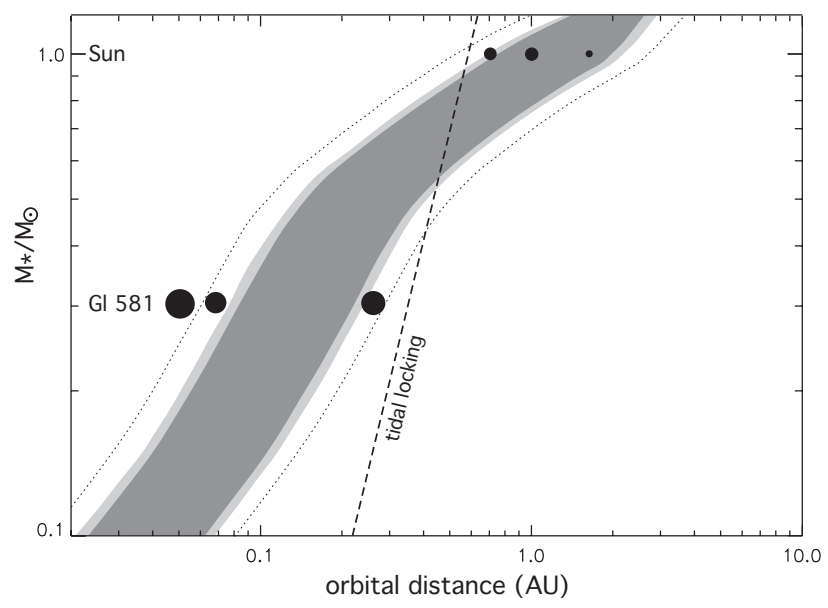

Fig. 3. The orbital region that remains continuously habitable during at least $5 \mathrm{Gyr}$ as a function of the stellar mass. The darker area is defined by the empirical "early Mars" and "recent Venus" criteria. The light grey region gives the theoretical inner (runaway greenhouse) and outer limits with $50 \%$ cloudiness, with $\mathrm{H}_{2} \mathrm{O}$ and $\mathrm{CO}_{2}$ clouds, respectively. The dotted boundaries correspond to the extreme theoretical limits, found with a $100 \%$ cloud cover. The dashed line indicates the distance at which a $1 M_{\oplus}$ planet on a circular orbit becomes tidally locked in less than $1 \mathrm{Gyr}$.

and (3) cannot be applied for $T_{\text {eff }}<3700 \mathrm{~K}$ because no radiativeconvective simulation has been performed for such low stellar effective temperatures. However, the albedo calculated for $T_{\text {eff }}=3700 \mathrm{~K}$ and $3200 \mathrm{~K}$ should be similar. Figure 4 shows the location of the boundaries of the HZ around Gl 581 for various criteria, along with the three known planets. The limits are given with or without the albedo correction (except for the cloud-free simulation, which was specifically run for $T_{\text {eff }}=3700 \mathrm{~K}$ ).

\subsection{The planet $\mathrm{GI} 581 \mathrm{C}$}

Figure 4 shows that planet Gl 581c would be habitable only if clouds with the highest reflectivity covered most of the daytime hemisphere. A 50\% cloud cover is not enough to prevent a runaway greenhouse effect on G1 581c, which receives $30 \%$ more energy than Venus today. This problem is exacerbated by the fact that Venus has a much higher albedo than the expected value for a habitable planet at the orbital distance of Gl 581c. The composition of the atmosphere of Gl 581c depends on the mass of the initial water reservoir on the planet, and on the efficiency of the gravitational escape of $\mathrm{H}$. Two possible scenarios, inspired by the fate of Venus and by simulations done for an Earth-like planets, can be suggested:

i) The reservoir of water is large so only a fraction of it will be lost to space. Numerical simulations suggest that planets more massive than about $5 M_{\oplus}$, such as those found around Gl 581 or HD 69830 (Alibert et al. 2006), started their formation in the cold outer protoplanetary disk, accreting some icy planetesimals, and migrated close to the star. In this situation, water can be orders of magnitude more abundant than on Earth. If this is the case for Gl 581c, its rocky surface is probably covered by a thick layer of $\mathrm{H}_{2} \mathrm{O}$. Depending on the amount, water can form a mantle of hot and high-pressure ice underneath a fluid envelope of supercritical $\mathrm{H}_{2} \mathrm{O}$ (Selsis et al. 2007). 


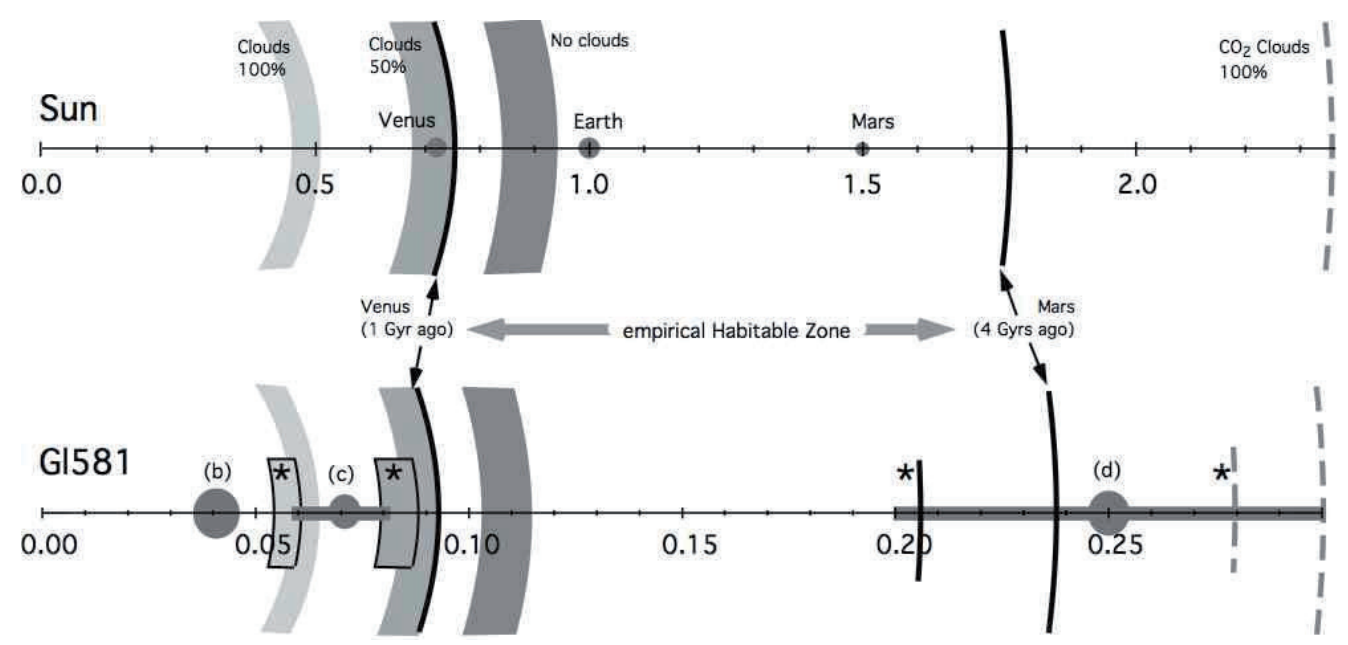

Fig. 4. Diagrams depicting the HZ around the Sun and Gliese 581. The grey areas indicate the theoretical inner edge for different fractional cloud covers. The width of each inner edge is defined by the runaway greenhouse and water loss limits. The thick lines give the inner and outer boundaries of the "empirical" HZ, based on the non-habitability of Venus for the last 1 Gyr and the apparent habitability of Mars 4 Gyr ago. The dashed line gives the outermost theoretical limit of the $\mathrm{HZ}$, found with a $100 \% \mathrm{CO}_{2}$ cloud cover. The upper diagram shows the limits computed for the Sun's properties. The lower diagram shows the limits computed for a $3700 \mathrm{~K}$ M-type star and scaled to the stellar luminosity of Gl 581 (for which $T_{\text {eff }}=3200 \mathrm{~K}$ ). The limits marked with the symbol $*$ are simply scaled to the luminosity and are thus slightly closer to the star - they are shown here because the wavelength dependency of the albedo has been explicitly computed only for thick and cloud-free $\mathrm{H}_{2} \mathrm{O}$ - and $\mathrm{CO}_{2}$-rich atmospheres. The albedo should be less sensitive to the stellar effective temperature in the presence of clouds. For the eccentric solution found by Udry et al. (2007), the orbits are confined within the grey bars about the marked planet locations.

ii) The reservoir of $\mathrm{H}_{2} \mathrm{O}$ has been lost earlier, when the star was still a strong X-ray and EUV emitter. $\mathrm{A} \mathrm{CO}_{2}$-rich, Venus-like atmosphere could remain, with surface temperatures still in the range $700-1000 \mathrm{~K}$. A significant level of leftover $\mathrm{O}_{2}$ may be present.

But the planet could also have a bulk composition that is so different from Earth that a broader diversity of situations should be explored. The planet could, for instance, be a volatile-rich planet (Kuchner 2003; Léger et al. 2004) or a carbon- or ceramic-planet (Kuchner \& Seager 2005; Gaidos 2000), with an atmospheric composition very different from our assumptions derived from the study of Venus, Earth, and Mars. However, water is considered an indispensable prerequisite for life and any additional atmospheric constituent would likely enhance the opacity of the atmosphere and the amount of greenhouse warming. The inner edge determined for a pure $\mathrm{H}_{2} \mathrm{O}$ atmosphere thus seems to be the closest possible limit for habitability.

\subsubsection{The effect of gravity}

The gravity at the surface of G1 581c, calculated for its minimum mass and for the range of radii predicted by Sotin et al. (2007) or Valencia et al. (2007), is between 1.3 and $2 \mathrm{~g}$. Therefore, a given value of the vapor pressure $P_{\mathrm{w}}\left(T_{\mathrm{s}}\right)$ would correspond to a vapor column 1.3-2.0 times smaller than the equivalent at Earth gravity. As the water column determines the IR opacity of the atmosphere, a higher gravity is expected to shift the inner edge of the HZ closer to the star. However, IR opacity is not the only parameter to be affected by the gravity. For a given value of $T_{\mathrm{s}}$, gravity also influences the lapse rate and the albedo produced by the water column. When treated self-consistently, these effects tend to compensate for each other, thus weakening the overall influence of gravity. Radiative-convective simulations of a planet with a surface gravity of $2.5 \mathrm{~g}$ were carried out by Kasting et al. (1993), who found an inner edge only 3\% closer. For the expected gravity of Gl 581c, its effect can be safely neglected.

\subsubsection{Stability of a quasi-snowball state}

For a given stellar irradiation, there is not necessarily a unique solution for $T_{\mathrm{s}}$. On Earth, for instance, climate is known to be bistable. It has been warm during most of its history, with a mean surface temperature well above $273 \mathrm{~K}$ (which includes glacial and interglacial periods), except for a few snowball Earth events characterized by an ice cover down to the equator. The most recent snowball events occurred at the end of Neoproterozoic era between 730 and $610 \mathrm{Myr}$ ago. At the beginning of these events, the runaway ice-albedo feedback makes the global mean temperature drop to $\sim 220 \mathrm{~K}$ for a few tens of thousands of years. This temperature drop is followed by a period of a few million years with a mean temperature around $265 \mathrm{~K}$ (Schrag et al. 2002). Thanks to the volcanic release of $\mathrm{CO}_{2}$ and the inefficiency of carbonate formation on a frozen Earth, our planet did not remain trapped in such state. This constitutes strong evidence of the long-term stabilization of the climate through the carbonatesilicate cycle. Snowball events illustrate the fact that the Earth would not be close enough to the Sun to maintain $T_{\mathrm{s}}$ above $273 \mathrm{~K}$ if water vapor was the unique greenhouse gas in the atmosphere. Without enough atmospheric $\mathrm{CO}_{2}$, the surface of our planet would be frozen, and the very high albedo of the frozen oceans would permanently keep $T_{\mathrm{s}}$ as low as $220 \mathrm{~K}$.

It can be considered whether such bistability could be possible on G1 581c. Radiative-convective models give one solution for the atmosphere structure and surface temperature, but would a cold solution be possible? Let us assume that ice covers the entire planetary surface, resulting in an albedo of $\sim 0.8$, and that the atmosphere has a negligible radiative effect, either because it is very tenuous or because it is composed of IR-transparent gases like $\mathrm{N}_{2}$. In this case, $T_{\text {eq }}$ would be close to $235 \mathrm{~K}$, which is consistent with this "snowball" hypothesis. However, in the absence of a greenhouse effect, and in the case of a slowly rotating planet (see Sect. 3.5), the surface temperature at the substellar point would be $330 \mathrm{~K}$. This temperature corresponds to a vapor pressure of 0.2 bar, which is inconsistent with the assumed albedo 
and atmospheric transparency. If we assume an extreme albedo of 0.95 , as on Enceladus, the icy satellite of Saturn, the substellar temperature would be well below $273 \mathrm{~K}$, and the icy state would be stable. However, in the absence of liquid water on the surface, the planet would not be habitable. An interesting situation might be found for a very narrow range of albedo $(A=0.89-0.90)$ for which most of the planetary surface is frozen, except for an area at the substellar point, where the local equilibrium temperature and the vapor pressure slightly exceed $273 \mathrm{~K}$ and 6.1 mbar, respectively. This would allow for the presence of liquid water.

In this latter case, though, the IR opacity and effect on the albedo of a 6.1 mbar vapor column is already important (in fact the mean water content of Earth's atmosphere is very close to this value) and is not consistent with the assumed surface temperature. This means that temperature and vapor pressure are expected to diverge rapidly from this assumed starting point. It would be interesting to study this case with a time-dependent model including the vaporization of water at the substellar point and its condensation in the cold regions, to check whether a substellar hot spot can exist without triggering a runaway greenhouse. Such a model should also include the rotation of the planet and the variation in the orbital distance due to the eccentricity. Indeed, a partially habitable steady-state implies that the substellar point moves on the planetary surface (otherwise all the water inventory ends up in the frozen regions), which requires significant eccentricity (see Sect. 3.5). However, this case is ad hoc as there is no particular reason why the planetary albedo would have this exact value and, if the planet was mainly frozen, any volcanic release of $\mathrm{CO}_{2}$ (expected on a planet as massive as G1 581c) would trigger a runaway greenhouse.

\subsection{The planet $\mathrm{Gl} 581 d$}

Planet Gl 581d receives about half the energy flux that Mars gets currently from the Sun. However, because $\mathrm{CO}_{2}$-rich atmospheres absorb more energy from an M-type star than from a G-type star, the orbital distance of Gl 581c is no more than $4 \%$ beyond the empirical "early Mars" limit (when scaled to Gl 581). Moreover, a partial cover of $\mathrm{CO}_{2}$-ice clouds can theoretically sustain habitable conditions for even lower stellar fluxes. The third planet of this system is thus potentially habitable, according to our present knowledge. Its high mass makes the maintenance of a thick atmosphere possible over billions of years. Of course, the high $\left(\sim 8 M_{\oplus}\right)$ minimum mass also means that the actual mass could be $>10 M_{\oplus}$, and the planet may thus be a gas or ice giant, rather than a rocky planet. If so, this planet is only one of the many other gas giant exoplanets already known to be within the HZs of their parent stars.

The warming effect of $\mathrm{CO}_{2}$-ice clouds has only been studied for Sun-like irradiation. Because of their size and their optical constants, typical $\mathrm{CO}_{2}$-ice particles are relatively transparent to visible radiation, but scatter efficiently at mid-IR wavelengths. For a stellar flux shifted towards the NIR, the albedo of these clouds could be significantly higher than for solar irradiation. This effect is not included in Eq. (3). Detailed modeling is thus required, without which it is not possible to determine the precise location of the edge of the $\mathrm{HZ}$ as defined from $\mathrm{CO}_{2}$-ice clouds, and thus the potential habitability of the large terrestrial planet Gl 581d.

As discussed in Sect. 2.3.3, some atmospheric compositions with the right greenhouse cocktail might provide stronger greenhouse warming than $\mathrm{CO}_{2}$ alone and sustain liquid water at the surface of G1 581d. In addition, some greenhouse gases, such as $\mathrm{CH}_{4}$, condense at much lower temperatures than $\mathrm{CO}_{2}$ (the triple point of $\mathrm{CH}_{4}$ is at $90 \mathrm{~K}$ and 0.1 bar) and would not be trapped as ice on the night side in case of synchronous rotation (see Sect. 3.5). It is not obvious that $\mathrm{CH}_{4}$ alone could maintain habitable conditions on Gl 581d. Indeed, the efficiency of the surface warming is expected to decrease above a certain $\mathrm{CH}_{4}$ level. This is due to the stratospheric heating by the direct absorption of the visible and near-IR stellar flux. For Sun-like irradiation and partial pressures of $\mathrm{CH}_{4}$ above about 5 mbars, this effect competes with the surface greenhouse warming. The temperature structure of a $\mathrm{CH}_{4}$-rich atmosphere under the irradiation of the star G1 581 should thus be specifically calculated to answer this question. A remaining problem is to identify the geochemical mechanisms able to stabilize a $\mathrm{CH}_{4}$-rich or a "super-greenhouse" atmosphere over several Gyr.

\subsection{An excess of volatiles?}

The volatiles that constitute the atmosphere of terrestrial planets in the Solar System have been mainly accreted as solids (rocks, with a possible minor contribution from ices). This is inferred from the composition in noble gases and their isotopes (Pepin 1991). If a significant mass of $\mathrm{H}_{2}$ and $\mathrm{He}$ was accreted directly from the protoplanetary nebula, most of it was lost to space and the traces that remain represent a negligible fraction of the present atmospheres. The last phases of the accretion of Earth are likely to have occurred after the dissipation of the protosolar nebula. Indeed, disks are found to typically live less than 5-10 Myr (Meyer et al. 2007), while it should have taken more than about 30 Myr to form the Earth (Martin et al. 2006). However, due to the diversity of disk properties (in lifetime, mass, density) and the role of migration (which accelerates the accretion), it is possible that the accretion of nebular gas is much more efficient on some terrestrial proto-exoplanets, especially if they are more massive than Earth. Rafikov (2006) found that planets more massive than $6 M_{\oplus}$ could have accreted a significant fraction of their mass as $\mathrm{H}_{2}$-He. For instance, a $8 M_{\oplus}$ planet could be made of $7 M_{\oplus}$ of rocks and $1 M_{\oplus}$ of $\mathrm{H}_{2}-\mathrm{He}$. The dissipation of the disk could have frustrated the evolution of such planet into a gas giant. It is thus possible that some planets more massive than $\sim 6 M_{\oplus}$ (which is potentially the case of Gl 581c and G1 581 d) could not host liquid water because of the pressure and temperature imposed by a massive envelope of gas. This situation would be similar to that of HD 69830d. This $18 M_{\oplus}$ planet found in the $\mathrm{HZ}$ of its star (Lovis et al. 2006) is thought to have accreted a massive $\mathrm{H}_{2}-\mathrm{He}$ envelope, underneath which water can only exist as a supercritical fluid or high-pressure ice (Alibert et al. 2006).

As mentioned before, the Gl 581 planets may very well have started their formation beyond the snow line. In this case, and even without invoking the accretion of hydrogen-rich gas from the protoplanetary nebula, their volatile content could be orders of magnitude higher than on Earth. If only $10 \%$ of the accreted solids are made of cometary ice, a $6 M_{\oplus}$ planet would contain as much as $0.55 M_{\oplus}$ of water and $0.06 M_{\oplus}$ of other volatiles, mainly $\mathrm{CO}_{2}, \mathrm{CO}, \mathrm{CH}_{4}$, and $\mathrm{NH}_{3}$. For comparison the mass of the terrestrial oceans is $2 \times 10^{-4} M_{\oplus}$ and the mass of Earth's atmosphere is $9 \times 10^{-7} M_{\oplus}$.

The distribution of these volatiles between the interior and the atmosphere (including here surface ices) at the end of the accretion depends on the planet's thermal history. Also, the volatiles initially present in the planetesimals may not be retained during the violent accretional collisions (Lissauer 2007). However, the planet can possibly have a massive and enriched 
gaseous envelope preventing the liquid phase of water from existing on the surface.

\subsection{Atmospheric erosion and the habitability of planets around M-type stars}

Models presented in this paper assume that planets can retain a dense atmosphere. However, planets in the HZ of active stars can be exposed to high levels of X-ray and EUV radiation (XUV) and strong particle fluxes from the quiescent stellar wind or coronal mass ejections (CMEs). Such high emissions are the result of the stellar magnetic activity and can induce important thermal and non-thermal atmospheric losses to space, potentially able to strip the whole atmosphere (Grießmeier et al. 2005; Lammer et al. 2006; Lammer 2007; Khodachenko et al. 2007; Lammer et al. 2007; Scalo et al. 2007). Within the HZ of the Sun and solar-type stars, conditions threatening the survival of the atmosphere and the habitability are limited to the first few hundred million years (Kulikov et al. 2007), and we know that the Earth and Venus atmospheres survived this early active phase (more damage may have been caused to Mars). But extreme irradiation conditions could last several Gyr in the case of M-type stars, as discussed below. In the context of Gl 581, it is thus worth addressing the question of stellar activity and the impact on the habitability of its planets.

For an initial estimate of the evolution of XUV irradiances we have used a proxy indicator, which is the ratio of the X-ray luminosity to the bolometric luminosity $\left(\log \left[L_{\mathrm{X}} / L_{\mathrm{bol}}\right]\right)$. This ratio is highest for the most active stars (i.e. fastest rotation period) and decreases monotonically with decreasing level of chromospheric activity (e.g., Stelzer \& Neuhäuser 2001; Pizzolato et al. 2003). From the analysis of open-cluster stars it is now well established that all single late-type stars (G-K-M) spin down as they age. Their activity decreases with time, and so does the ratio $\log \left(L_{\mathrm{X}} / L_{\mathrm{bol}}\right)$. It is also a well-known effect that $\log \left(L_{\mathrm{X}} / L_{\mathrm{bol}}\right)$ does not increase up to values arbitrarily close to unity for very active stars. Instead, a "saturation" phenomenon occurs and no active star seems to go higher (except for the strongest flares) than $\log \left(L_{\mathrm{X}} / L_{\mathrm{bol}}\right) \approx-3$ (e.g., Vilhu \& Walter 1987; Stauffer et al. 1994). Qualitatively, the evolution of $\log \left(L_{X} / L_{\text {bol }}\right)$ for a late-type star has a flat plateau from its arrival on the main sequence up to a certain age (end of saturation phase) and then decreases monotonically as a power law function of age.

We have compiled a sample of $\mathrm{K}$ - and M-type stars with ages determined in a similar way to the solar analogs in the "Sun in Time" sample (Ribas et al. 2005), i.e., membership in clusters and moving groups (Montes et al. 2001), rotation period, membership in wide binaries, and isochrones. For these, $\log \left(L_{\mathrm{X}} / L_{\mathrm{bol}}\right)$ values have been obtained from the thorough list provided by Pizzolato et al. (2003) and complemented in a few cases with values estimated directly from ROSAT measurements following Schmitt et al. (1995). The evolution of $\log \left(L_{X} / L_{\text {bol }}\right)$ with age for stars of different spectral types is illustrated in Fig. 5. Note that today's Sun has a value of $\log \left(L_{\mathrm{X}} / L_{\mathrm{bol}}\right) \sim-6.1$. The solid lines are semi-empirical estimates that result from the fact that the evolution of $L_{X}$ in the power law regime is roughly independent of the spectral type and $L_{\mathrm{bol}}$ comes from the use of stellar models for different masses. For the K- and M-type stars these are preliminary estimates and the uncertainty of each point can be of a few tenths of a dex. In the case of G-type stars, the values plotted are much more reliable and come from direct measurements for the "Sun in Time" targets. More details on this analysis will be given in a forthcoming publication (Ribas et al. 2008, in prep.).

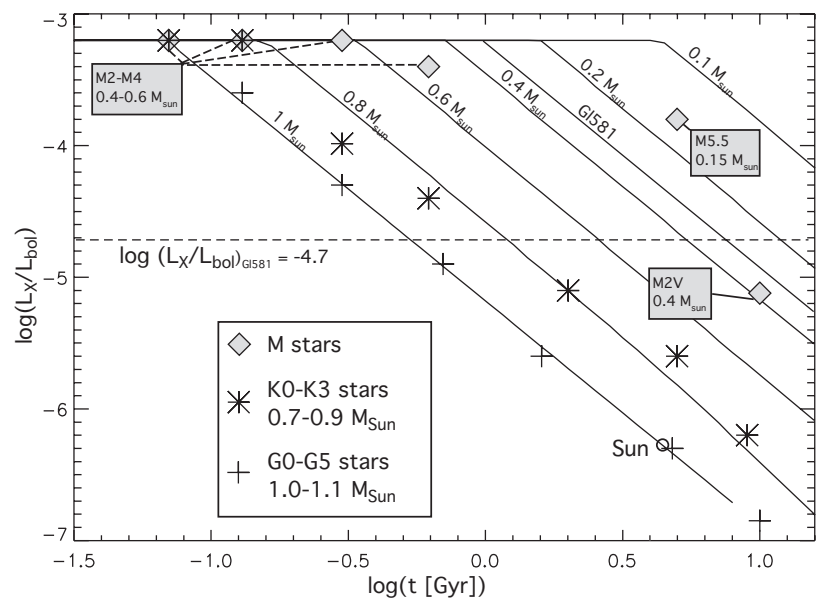

Fig. 5. Evolution of the ratio between the X-ray and bolometric luminosities as a function of age for stars of different masses. The solid lines represent semi-empirical laws, while symbols give observed values for $\mathrm{G}(+), \mathrm{K}(*)$ and $\mathrm{M}(\diamond)$ stars. The dashed line gives the upper limit for the value of $\log \left(L_{\mathrm{X}} / L_{\mathrm{bol}}\right)$ in the case of Gl 581, for which there is no ROSAT detection.

It is reasonable to assume that the overall XUV flux received by a planet in the $\mathrm{HZ}$ scales with the ratio $\log \left(L_{\mathrm{XUV}} / L_{\mathrm{bol}}\right)$. Figure 5 shows that, while solar-type stars stay at saturated emission levels until ages of $\sim 100$ Myr and then their XUV luminosities rapidly decrease following a power-law relationship as a function of age, M-type stars have saturated emissions (i.e., highest activity) up to ayes of a few Gyr. Thus, planets in the HZ of M-type stars may receive XUV fluxes that are 10-100 times higher than in the $\mathrm{HZ}$ of solar-type stars of the same age.

To estimate the possible activity level of Gl 581 we carried out a search in the ROSAT Faint Source Catalog (Voges et al. 2000) that produced no result. Thus, Gl 581's X-ray flux is below the ROSAT detection threshold. Such a threshold can be calculated following Schmitt et al. (1995) and we found that $L_{\mathrm{X}}$ must be lower than $10^{27} \mathrm{erg} \mathrm{s}^{-1}$ at the distance of Gl 581, implying that $\log \left(L_{\mathrm{X}} / L_{\mathrm{bol}}\right)<-4.7$. From Fig. 5, this leads to a lower limit of the age that, considering the associated uncertainties, could be around $7 \mathrm{Gyr}$. However, we caution that this is still a preliminary estimate and that Gl 581 should be studied further before giving a conclusive figure. With regards to an upper limit to the age, the space motions of Gl $581(U, V, W)=(-22.7,-29.3,-8.0) \mathrm{km} \mathrm{s}^{-1}$ are characteristic of a galactic disk star, and thus its age should not be above 10-11 Gyr. Also, the star is known to have a only midly subsolar metallicity $([\mathrm{Fe} / \mathrm{H}] \approx-0.3$; Bonfils et al. 2005; Bean et al. 2006), which is also consistent with being a disk member.

The current XUV emissions of Gl 581 may not represent a significant threat to the stability of planetary atmospheres inside the HZ, but these planets have most likely suffered strong erosion phenomena during earlier stages. Dominant loss mechanisms are thermal escape, induced by the heating of the upper atmospheric layers by XUV irradiation, and non-thermal escape caused by the interaction of these upper layers with the stellar particle wind. The efficiency of these erosion processes depends on several factors. First, the composition of the atmosphere controls the temperature of the exosphere and thus the thermal escape rates, which can be extremely high if the blow-off temperature is reached (Lammer et al. 2006). A high mixing ratio of $\mathrm{CO}_{2}$, for instance, allows the exosphere to cool efficiently by IR emission (that is why the exosphere of Venus is about three times cooler than Earth's). The thermal structure of the upper 
atmosphere also determines the extension of the exosphere and indirectly the efficiency of non-thermal losses (like sputtering, ion-pick up, dissociative recombination). Non-thermal losses also depend on poorly constrained parameters like the intrinsic magnetic moment of the planet and on the stellar wind properties (velocity and density).

Observational constraints on stellar winds exist (Wood et al. 2005), but their interpretation is still debated (Holzwarth \& Jardine 2007). It does however seem well established that the particle winds of active M-type stars could at least be 10 times stronger than that of today's Sun, in the HZ. During the early and active stages of an M-type star like Gl 581, the rate of atmospheric losses due to the association of XUV heating and particle wind-induced ion pick-up could theoretically reach tens of bars of heavy elements $(\mathrm{O}, \mathrm{C}, \mathrm{N})$ per Gyr for a weakly-magnetized planet in the $\mathrm{HZ}$, even with a $\mathrm{CO}_{2}$-rich atmosphere (Lammer et al. 2007). Tidal interactions rapidly slow down the rotation of planets in the HZ of M-type stars (see Sect. 3.5), which may result in weak intrinsic magnetic moments, unable to provide sufficient protection against non-thermal erosion. On the other hand, Gl 581c and Gl 581d are significantly more massive than the Earth, which may result in an enhanced dynamo and better shielding. Their surface gravities are also higher by factors 1.3 and 2.5, respectively, which limits the extension of the exosphere and should lower the erosion rate.

Eventually, the threat posed by the atmospheric erosion depends on the inventory of volatiles, as has already been discussed in the case of the water loss in Sect. 2.2.2. If the loss rate exceeds the outgassing rate, or if the atmospheric losses occurring during the planet's early stages exhaust species essential for the maintenance of life, the planet would obviously not remain habitable in the long term. Gaidos \& Selsis (2007) point out that a replica of early Earth (assumed to have a $\mathrm{CO}_{2}$-rich atmosphere) located in the HZ of a M-type star could lose its entire atmospheric nitrogen inventory during its first Gyr via thermal escape from XUV heating alone. A large reservoir of volatiles and/or a continuous outgassing of the essential atmospheric species is thus required to ensure durable habitable conditions around M-type stars.

Models providing quantitative estimates for atmospheric escape in the extreme conditions encountered in the HZ of M stars are still under development. Available preliminary results cited in this section are extremely promising and stress the importance of atmospheric retention for habitability around low-mass stars. However, current estimates of thermal and non-thermal losses in this context assume a static upper atmosphere, while high atmospheric escape rates would require a hydrodynamic treatment. This field of research has recently become very active due to the important questions raised by the habitability of planets around M-type stars. Although we have to end this section with a question mark, the reader should expect forthcoming results obtained with thermal hydrodynamic models coupled with non-thermal erosion that will shed more light on the fate of planetary atmospheres around active stars.

\subsection{The effect of rotation and eccentricity}

Udry et al. (2007) present two fits of the radial velocity curve, one obtained with fixed circular orbits, and another where the eccentricity is a free parameter. The latter resulted in the best agreement and yielded $e=0.16$ and $e=0.2$ for G1 581c and Gl 581d, respectively.

If the orbits are circular, then these planets could have evolved into a synchronized state, in which the rotation period $\left(P_{\mathrm{r}}\right)$ equals the orbital period $(P)$. The synchronization time of these planets is indeed very short. We obtain $25000 \mathrm{yr}$ and $10^{7} \mathrm{yr}$ for planets G1 581c and Gl 581d, respectively, when adopting a value of $Q=100$ for the dissipation factor, typical of terrestrial planets. In this case, the planets have permanent day and night hemispheres. It is interesting to note that we find a much longer circularization time for these two planets $\left(10^{10} \mathrm{yr}\right)$, which is consistent with eccentric orbits. If the planets do have a significant eccentricity, then the following cases are possible:

i) Because terrestrial planets are expected to have a non purely axisymmetric shape, tidal spin down may have made it possible for the rotation to be captured in a spin-orbit resonance with $P=(n / 2) P_{r}$. Mercury, for instance, is trapped in a 3:2 spin-orbit resonance as a consequence of its oblong shape. For resonances with $n \neq 2$, the star rises and sets at any point of the planet and there is no permanent dark area. In the synchronous case $(n=2)$, there is a region of the planet that never receives direct starlight. For an eccentric planet with zero obliquity, the size of the corresponding surface is governed by the amplitude of the optical librations about the substellar point when neglecting the smaller contribution of forced and free librations. Considering up to thirdorder terms in eccentricity, we estimate that the amplitude of the libration is $2 e+25 e^{3} / 16$, and thus the fractional area $r$ of the planet that can receive direct starlight, is then given by

$r=\frac{1}{2}+\frac{2 e+25 e^{3} / 16}{\pi}$.

For $e=0.2$, the optical libration amounts to $\sim 23^{\circ}$, and $r$ would take a value of $63 \%$. Any non-zero obliquity adds latitudinal librations that may significantly increase these values. For high obliquities, it is possible for the entire surface of the planet to receive direct starlight some time during an orbital period. However, high obliquities are not consistent with tidally-driven evolution models of the spin axis.

ii) The planet has not been captured in a spin-orbit resonance. In this case, no region of the planet is permanently dark.

Let us now consider the synchronized case of $P_{\mathrm{r}}=P$, with low obliquity. Whether the orbit is eccentric or not, there is a region on the planet that is not directly heated by the stellar radiation. Such a region can be an entire hemisphere if the eccentricity is negligible. There is thus a risk of the atmospheric constituent condensing irreversibly on an ice sheet covering the dark region. Joshi et al. (1998) and Joshi (2003) have shown that the atmosphere has to be sufficiently dense and opaque in the IR to transport enough heat to the dark area and to prevent such an atmospheric collapse. Near the edges of the HZ, a habitable planet fulfills these conditions because it is very rich in either $\mathrm{H}_{2} \mathrm{O}$ or $\mathrm{CO}_{2}$. Therefore, if one concludes that planets $\mathrm{Gl} 581 \mathrm{c}$ and Gl 581d are habitable thanks to the effect of clouds, then synchronized rotation would not appear to represent an impossible obstacle to their habitability (although this would give rise to a climate that is certainly not "Earth like", with a super-rotating atmosphere). In addition, the strong irradiation of planet Gl 581c is expected to produce strong thermal tides in the atmosphere (similar to those on Venus) that could prevent the synchronization of the planet (Correia \& Laskar 2003).

For planet Gl 581d, whose orbit is in the outer area of the HZ, synchronization can pose a more significant threat. The habitability of the surface of the Earth has remained constant during most of its history, except for a few snowball events possibly generated by climatic instabilities. The Earth was able to recover from these frozen episodes thanks to the continuous release of 
volcanic $\mathrm{CO}_{2}$. However, a synchronized planet may not be able to recover from a snowball event as effectively as the Earth did because the condensation of $\mathrm{CO}_{2}$ on the dark side would occur much faster than the release of volcanic $\mathrm{CO}_{2}$. Only a rapid and dramatic event, such as a large impact on the ice sheet, could initiate a new habitable period. The habitable state of a synchronized planet could thus be particularly fragile when it relies on the presence of atmospheric $\mathrm{CO}_{2}$, which is the case for most of the $\mathrm{HZ}$ except for the very narrow inner part where $\mathrm{H}_{2} \mathrm{O}$ is "self sufficient".

Long-term habitability on a synchronized planet could still be maintained if the greenhouse warming was provided by atmospheric compounds that remain gaseous at sufficiently low temperature, such as $\mathrm{CH}_{4}$. In this situation, one may wonder if an inefficient transport of the incident energy from the starlit to the dark hemisphere could help maintain habitable conditions on the starlit hemisphere, or in a smaller region around the substellar point, while the dark hemisphere would remain too cold to host liquid water. In this case, restricting the habitability to a fraction of the planetary surface would compensate for the low stellar flux. Although this case represents a tantalizing configuration, the cold trap for water would still operate, and this would be irreversibly transported from the habitable to the frozen region.

In the case of the planets having an eccentricity as high as $0.16-0.2$, the study of the climate at steady state is of course less relevant than for a circular orbit. As shown by Fig. 4, both Gl 581c and Gl 581d are likely to make incursions inside the $\mathrm{HZ}$ (or excursions outside the HZ) depending on the assumed boundaries. This situation is not expected to change the discussion significantly regarding planet Gl 581c, since the timescales associated with the vaporization of the water reservoir are significantly longer than the 12-d orbital period. In addition, Williams \& Pollard (2002) show that habitability depends primarily on the average stellar flux received over an entire orbit, $\langle S\rangle$, even at high eccentricity, and $\langle S\rangle$ depends on the eccentricity through the relationship:

$$
\langle S\rangle=\frac{S}{\sqrt{1-e^{2}}}
$$

where $S$ is the stellar flux at the semi-major axis of the orbit. For $e=0.16$ and $e=0.20$ the average stellar flux is greater by $1.3 \%$ and $2.1 \%$, respectively, in comparison with a circular orbit. With G1 581d located near the outer edge of the HZ, such slightly higher flux may be precious for the maintenance of habitability. Excursions out of the HZ may, however, increase the efficiency of irreversible condensation on the dark side, although these excursions could last only a fraction of the $83-\mathrm{d}$ period.

\subsection{Internal heat flux and tidal dissipation}

Geothermal heat has a major impact on climate during the early phases of planetary evolution. Both during and shortly after accretion, it can trigger a runaway greenhouse and a surface magma ocean provided that the cooling to space is limited by a dense atmosphere, which would be blanketing the thermal emission (Abe \& Matsui 1988). This is due to the large amount of energy released in a short period of time and to an efficient convective transport of internal energy to the surface by a yet liquid mantle. The period during which Earth's surface was significantly heated "from underneath" was restricted to the first tens of Myr. On the present Earth, the internal heat flux, dominated by the decay of radiogenic species and the release of the initial accretion energy, is about 5000 times lower than the solar energy absorbed by the planet. Thus, the direct influence of the internal heat on the climate has been negligible for the past $4.50 \mathrm{Gyr}$ or so. Similarly, the Gl 581 system is most likely too old (see Sect. 3.4) to invoke geothermal forcing of the climate.

The difference in mass and radius between the Earth and the G1 581 planets should not affect this reasoning significantly. The mean flux of accretion energy through the surface scales with $M^{2} / R^{3}$ and remains within 10 times that of the Earth. Assuming the same abundance of radiogenic species for the Gl 581 planets, the associated heat flux should scale with $M / R^{2}$ and remain Earth-like within a factor of $\sim 2$. Only by assuming orders of magnitude more radiogenic species per unit mass could the geothermal flux be significant for the global energy balance on the surface.

Interestingly, during the few Myr after the Moon-forming impact, the tidal interaction with the Moon might have been a major heating source for Earth's climate (Zahnle et al. 2007). Tidal dissipation was favored by the small Earth-Moon distance and most of the dissipated energy was taken from the kinetic rotation energy. Because the rotation of Gl 581 planets is presumed to be tidally evolved, tidal dissipation may still occur but through the damping of their high eccentricity and may increase the internal heat flux to a level that could affect the climate. It is thus worth exploring this point further.

Assuming synchronously rotating and eccentric planets, and using formulae from Peale (1999) with a tidal dissipation factor $Q=100$, we find that $1.4 \times 10^{16} \mathrm{~W}$ and $3.1 \times 10^{12} \mathrm{~W}$ are dissipated respectively in planets Gl 581c and Gl 581d (these values are obtained with the largest radii given in Table 1 to analyze the maximum effect). This dissipated power corresponds to a maximum surface flux of $10 \mathrm{~W} \mathrm{~m}^{-2}$ for planet $\mathrm{Gl} 581 \mathrm{c}$ and $0.0015 \mathrm{~W} \mathrm{~m}^{-2}$ for planet Gl 581d.

On Gl 581c, tidal dissipation can contribute slightly to the energy balance, with the heat flux being potentially as high as $2 \%$ of the absorbed stellar energy (about $1 \%$ of the stellar flux at the planet's distance, averaged over the planetary surface). Besides this direct warming, this heat flux can increase the volcanic activity and the release of $\mathrm{CO}_{2}$. At the orbital distance of Gl 581c, additional $\mathrm{CO}_{2}$ can only further destabilize the climate against water loss or a runaway greenhouse. Therefore, tidal heating caused by a possibly non-zero eccentricity speaks against any habitable conditions on this planet.

On planet Gl 581d, tidal dissipation is negligible $(0.003 \%$ of the absorbed stellar energy) and cannot help to directly provide habitable conditions on its surface despite the low stellar flux. The enhanced heat flux (compared to that of the Earth) due to both the high planetary mass and the tidal heating might, however, have indirect effects. By inducing more volcanism and lasting longer over the planet's history, a higher heat flux favors the maintenance of the high $\mathrm{CO}_{2}$ level required on this planet. This may be important when taking into account that the system may be significantly older than the Earth.

\section{Discussion}

\subsection{Uncertainties in the location of the $H Z$ limits}

Different uncertainties affect the determination of the boundaries of the HZ. Recently released data on $\mathrm{H}_{2} \mathrm{O}$, such as the spectroscopy and equation of state at high pressure and the temperature and Rayleigh scattering cross-sections, could induce slight modifications on the planetary energy budget and should be included in radiative-convective models. Other data are still 
lacking, including detailed parameterization of the $\mathrm{CO}_{2}-\mathrm{CO}_{2}$ collision-induced absorption, which is an important factor for the greenhouse warming close to the outer limit of the HZ.

For $\mathrm{H}_{2} \mathrm{O}$ - and $\mathrm{CO}_{2}$-rich atmospheres, respectively at the inner and outer edge of the $\mathrm{HZ}$, the sensitivity of the planetary albedo on the stellar effective temperature, from which Eqs. (2) and (3) are derived, was calculated assuming a blackbody spectral distribution of the stellar flux. In reality, $\mathrm{TiO}$ and $\mathrm{H}_{2} \mathrm{O}$ absorption makes the spectrum of M-type stars quite different from a black-body. The calculation should be done for realistic spectra. For Gl 581, the expected effect would be to slightly move the $\mathrm{HZ}$ boundaries away from the star, because TiO bands mostly block visible light and shift the emission towards the IR. The albedo sensitivity to $T_{\text {eff }}$ should also be studied for cloudy atmospheres of planets. Since clouds are a crucial factor in the location of the $\mathrm{HZ}$ boundaries, future simulations will have to include the effect of the spectral type of the star, in particular for $\mathrm{CO}_{2}$ clouds whose properties are very sensitive to the wavelength of the incident flux.

An important step forward, especially in predicting the distribution of clouds, will be the use of 3D global climate models (GCMs), as was done to study synchronously rotating planets (Joshi et al. 1997; Joshi 2003) or to address the runaway greenhouse effect (Ishiwatari et al. 2002). These models will have to include realistic microphysical processes for the formation, growth, and destruction of droplets and icy particles. To reduce the computing time, the treatment of radiative transfer in GCMs has to be greatly simplified. This is currently the main limitation to the application of GCMs to exoplanets. At the moment, the detailed line-by-line modeling required to accurately describe the transfer of radiation in planetary atmospheres is restricted to $1 \mathrm{D}$ simulations. The development of 3D GCMs, including robust treatment of radiative transfer, cloud physics, and photochemistry, represents the future direction of theoretical studies of habitability.

\subsection{Geophysical influences}

In Von Bloh et al. (2007, hereafter VB07), the estimate of the surface temperature is done by solving a system of coupled equations, including many geophysical processes affecting the $\mathrm{CO}_{2}$ atmospheric level (such as weathering and outgassing rates) and a radiation balance equation (Eq. (4) of their paper), written as follows:

$$
\frac{L}{4 \pi d^{2}}\left[1-a\left(T_{\text {surf }}, P_{\mathrm{CO}_{2}}\right)\right]=4 I_{\mathrm{R}}\left(T_{\text {surf }}, P_{\mathrm{CO}_{2}}\right)
$$

where $a$ is the Bond albedo, $I_{\mathrm{R}}$ the outgoing thermal emission and $d$ the orbital distance. As shown in the present study, calculating $a$ and $I_{\mathrm{R}}$ requires detailed radiative-convective modeling and, besides their dependence on $T_{\text {surf }}$ and $P_{\mathrm{CO}_{2}}, a$ and $I_{\mathrm{R}}$ are highly sensitive to the spectral type of the star, the cloud properties and cover, and the abundance of other radiatively active atmospheric compounds. Solving Eq. (6) numerically requires adopting a simple mathematical form for $a\left(T_{\text {surf }}, P_{\mathrm{CO}_{2}}\right)$ and $I_{\mathrm{R}}\left(T_{\text {surf }}, P_{\mathrm{CO}_{2}}\right)$, for instance a fit of climate model results. This mathematical form and the climate models from which it derives are not given in VB07, but most likely they use the same method as the previous works of the group (e.g., Franck et al. 2002). This is based on an interpolation of radiativeconvective results obtained for specific cases by Kasting (1993) and Caldeira \& Kasting (1992) for a cloud-free atmosphere. This is consistent with the HZ inner limit shown in VB07 (Fig. 3b) corresponding (at $t=0 \mathrm{Gyr}$ ) to a radiative-convective calculation with $T_{\text {surf }}=100{ }^{\circ} \mathrm{C}$ and no clouds. There are several points we would like to discuss here:

i) A parameterization derived from Kasting et al. (1993) can only be applied close to the conditions where the full radiative-convective modeling is available, which is close to the inner edge of the $\mathrm{HZ}$. The radiative $\mathrm{HZ}$ would include Gl 581d only if the effect of $\mathrm{CO}_{2}$ clouds or another greenhouse gas is included. As there are only very few of these calculations at very low stellar irradiation, it would be very useful to know how $a$ and $I_{\mathrm{R}}$ are calculated in VB07, in particular close to the outer edge of the HZ.

ii) As shown in the present study, clouds have a major but uncertain effect on the location of both edges of the HZ. The inner edge, for instance, can be located anywhere between $0.05 \mathrm{AU}$ (runaway greenhouse with $100 \%$ cover of highly reflective clouds) and $0.11 \mathrm{AU}\left(100{ }^{\circ} \mathrm{C}\right.$, no clouds $)$ from the star G1 581. Therefore, the possible effects of clouds have to be compared with the geophysical influences included in VB07.

iii) Although the stellar luminosity is fixed in VB07 (which is a good approximation for $M$ stars), one can see in their Fig. 3 that the inner boundary of the $\mathrm{HZ}$ (fixed at $T_{\text {surf }}=100{ }^{\circ} \mathrm{C}$ ) moves outward as the planet evolves. Unfortunately, this effect is not explained in the paper, so we do not know what geophysical process is involved.

The present study and VB07 present two approaches that should complement each other. On one hand, we provide atmospheric models developed to compute the radiative transfer and atmospheric structure in a self-consistent way. On the other hand, VB07 use a box model including geophysical processes relevant for the maintenance of habitable conditions. Each of these processes has to be included in the VB07 model as a simple mathematical equation (e.g., Eq. (6)) to allow the numerical calculation of all the variables through a system of coupled equations. Although we fully recognize the potential usefulness of this approach, we would certainly appreciate an investigation of the sensitivity of the VB07 model to all the parameters involved and some estimation of the uncertainties affecting the model predictions.

The most noteworthy effect shown in VB07 and previous works from this group is the narrowing of the actual HZ compared to the radiative $\mathrm{HZ}$ presented for instance in our present study. The habitability of a planet can indeed be frustrated by the lack of outgassed $\mathrm{CO}_{2}$, as it may have occurred at some point in Mars' history. The age at which the decrease of internal heat and outgassing makes a planet no longer habitable obviously depends on the planetary mass and composition. For a given stellar type, age, and planetary mass, the VB07 model computes the location of the frontier between the inner region of the $\mathrm{HZ}$ that can actually be searched for habitable planets and the outer HZ region that can host only the dead remains of formerly habitable planets. However, to define this frontier accurately requires a robust model for each individual geophysical process, as well as strong constraints on the possible variety of planetary compositions; otherwise, we may rule out as "uninhabitable" circumstellar regions that could be prime targets in the search for habitable worlds.

Two recent publications reach opposite conclusions on the onset of plate tectonics on large terrestrial planets. According to Valencia et al. (2007a), plate tectonics become more likely as planets get bigger. O'Neill et al. (2007) come to the opposite conclusion that the higher gravity of big planets tends to 
prevent the formation of plates, producing a thick and unique crust. This debate illustrates the present difficulties in extrapolating Earth's geophysical models to exoplanets. Research on these important topics must of course keep going, but it is probably safer at this point to keep the broadest and less specific definition of the HZ boundaries from Kasting et al. (1993) ${ }^{1}$ and to allow future observations to determine whether a planet found within these boundaries actually is habitable.

\section{Conclusion: are GI 581c and GI 581d good targets for Darwin/TPF?}

According to our present knowledge, based on available models of planetary atmospheres, and assuming that the actual masses of the planets are the minimum masses inferred from radial velocity measurements, Gl 581c is very unlikely to be habitable, while Gl 581d could potentially host surface liquid water, just as early Mars did.

Because of the uncertainties in the precise location of the $\mathrm{HZ}$ boundaries, planets at the edge of what is thought to be the $\mathrm{HZ}$ are crucial targets for future observatories able to characterize their atmosphere. At the moment, our theory of habitability is only confirmed by the divergent fates of Venus and the Earth. We will have to confront our models with actual observations to better understand what makes a planet habitable. The current diversity of exoplanets (planets around pulsars, hot Jupiters, hot Neptunes, super-Earths, etc.) has already taught us that Nature has a lot more imagination when building a variety of worlds than we expected from our former models inspired by the Solar System.

It is obvious that the idealized model of a habitable planet atmosphere, where the two important constituents are $\mathrm{CO}_{2}$ and $\mathrm{H}_{2} \mathrm{O}, \mathrm{CO}_{2}$ being controlled by the carbonate-silicate cycle, is likely to represent only a fraction of the diversity of terrestrial planets that exist at habitable distances from their parent star. As an example, planets fully covered by an ocean may be common, either because they are richer in water than Earth or because the distribution between surface and mantle water is different, or perhaps simply because, for a given composition, the massto-surface ratio and thus the water-to-surface ratio increases with the planetary mass, as noted by Lissauer (1999). Without emerged continents, it is not at all clear that the carbonatesilicate cycle could operate. The planets around G1 581 can fall into this category since they are significantly more massive than the Earth (especially the $>8 M_{\oplus}$ planet Gl 581d) and also because they may have started their formation in the outer and more water-rich region of the protoplanetary disk.

Darwin/TPF-I and TPF-C could eventually reveal what the actual properties of the atmosphere of Gl 581c and Gl 581d are. From their thermal light curves we could infer if a thick atmosphere is making the climate more or less uniform on both the day and night hemispheres of these planets, despite a (nearly?) synchronized rotation (Selsis 2004). Visible and mid-IR water vapor bands could be searched in the atmosphere of Gl 581d to confirm its habitability. Mid-IR spectra of this planet could also reveal other greenhouse gases at work. Spectral observations of Gl 581c could potentially distinguish between a Venus-like atmosphere dominated by $\mathrm{CO}_{2}$ or an $\mathrm{H}_{2} \mathrm{O}$-rich atmosphere. The

1 The precise location of the HZ boundaries based on this definition can of course be revised to account for new molecular data, additional greenhouse agents, 3D calculations, or better cloud models as done, for instance, by Forget \& Pierrehumbert (1997), who estimated the possible effect of $\mathrm{CO}_{2}$ clouds in the location of the outer boundary. detection of $\mathrm{O}_{2}$ on this planet would generate a fascinating debate about its possible origin: as either a leftover of $\mathrm{H}_{2} \mathrm{O}$ photolysis and $\mathrm{H}$ escape or a biological release. There is certainly no doubt that Gl 581c and Gl 581d are prime targets for exoplanet characterization missions.

\section{References}

Abe, Y., \& Matsui, T. 1988, J. Atmosph. Sci., 45, 3081

Alibert, Y., Baraffe, I., Benz, W., et al. 2006, A\&A, 455, L25

Baraffe, I., Chabrier, G., Allard, F., \& Hauschildt, P. 1998, A\&A, 337, 403

Bean, J. L., Benedict, G. F., \& Endl, M. 2006, ApJ, 653, L65

Beaulieu, J.-P., Bennett, D. P., Fouqué, P., et al. 2006, Nature, 439, 437

Beichman, C. A., Woolf, N. J., \& Lindensmith, C. A. 1999, The Terrestrial Planet Finder (TPF): a NASA Origins program to search for habitable planets (JPL Publications)

Bibring, J.-P., Langevin, Y., Mustard, J. F., et al. 2006, Science, 312, 400

Bonfils, X., Forveille, T., Delfosse, X., et al. 2005, A\&A, 443, L15

Caldeira, K., \& Kasting, J. F. 1992, Nature, 360, 721

Correia, A. C. M., \& Laskar, J. 2003, Icarus, 163, 24

de Bergh, C., Bezard, B., Owen, T., et al. 1991, Science, 251, 547

Ehrenreich, D., Lecavelier des Etangs, A., Beaulieu, J.-P., \& Grasset, O. 2006, ApJ, 651, 535

Forget, F. 2007, Water and Climates on Mars, Lectures in Astrobiology, Advances in Astrobiology and Biogeophysics (Berlin, Heidelberg: SpringerVerlag), 103

Forget, F., \& Pierrehumbert, R. T. 1997, Science, 278, 1273

Franck, S., Kossacki, K. J., von Bloh, W., \& Bounama, C. 2002, Tellus Series B, Chem. Phys. Meteorol. B, 54, 325

Gaidos, E., \& Selsis, F. 2007, Protostars and Planets V, 929

Gaidos, E. J. 2000, Icarus, 145, 637

Gomes, R., Levison, H. F., Tsiganis, K., \& Morbidelli, A. 2005, Nature, 435, 466 Grießmeier, J.-M., Stadelmann, A., Motschmann, U., et al. 2005, Astrobiology, 5,587

Haqq-Misra, J. D., Domagal-Goldman, S. D., Kasting, P. J., \& Kasting, J. F. 2007 , in prep.

Hart, M. H. 1979, Icarus, 37, 351

Holzwarth, V., \& Jardine, M. 2007, A\&A, 463, 11

Ishiwatari, M., Takehiro, S.-I., Nakajima, K., \& Hayashi, Y.-Y. 2002, J. Atmosph. Sci., 59, 3223

Joshi, M. 2003, Astrobiology, 3, 415

Joshi, M. M., Haberle, R. M., \& Reynolds, R. T. 1997, Icarus, 129, 450

Kaltenegger, L., \& Fridlund, M. 2005, Adv. Space Res., 36, 1114

Kaltenegger, L., Traub, W. A., \& Jucks, K. W. 2007, ApJ, 658, 598

Kasting, J. F. 1988, Icarus, 74, 472

Kasting, J. F. 1991, Icarus, 94, 1

Kasting, J. F., \& Catling, D. 2003, ARA\&A, 41, 429

Kasting, J. F., Whitmire, D. P., \& Reynolds, R. T. 1993, Icarus, 101, 108

Khodachenko, M. L., Ribas, I., Lammer, H., et al. 2007, Astrobiology, 7, 167

Kuchner, M. J. 2003, ApJ, 596, L105

Kuchner, M. J., \& Seager, S. 2005, ArXiv Astrophysics e-prints

Kulikov, Y. N., Lammer, H., Lichtenegger, H. I. M., et al. 2007, Space Sci. Rev., 129,207

Lammer, H. 2007, Astrobiology, 7, 27

Lammer, H., Kulikov, Y. N., \& Lichtenegger, H. I. M. 2006, Space Sci. Rev., 122,189

Lammer, H., Lichtenegger, H. I. M., Kulikov, Y. N., et al. 2007, Astrobiology, 7, 185

Léger, A., Selsis, F., Sotin, C., et al. 2004, Icarus, 169, 499

Levine, H., Shaklan, S., \& Kasting, J. 2006, Terrestrial Planet Finder Coronagraph Science and Technology Definition Team (STDT) Report, Tech. rep. (Pasadena, CA: Jet Propulsion Laboratory)

Lissauer, J. J. 1999, Nature, 402, C11

Lissauer, J. J. 2007, ApJ, 660, L149

Lovis, C., Mayor, M., Pepe, F., et al. 2006, Nature, 441, 305

Lunine, J. I. 1999, Earth: evolution of a habitable world, ed. J. I. Lunine (Cambridge, UK: Cambridge University Press)

Martin, H., Albarède, F., Claeys, P., et al. 2006, Earth Moon and Planets, 98, 97 McKay, C. P., Toon, O. B., \& Kasting, J. F. 1991, Nature, 352, 489

Meyer, M. R., Backman, D. E., Weinberger, A. J., \& Wyatt, M. C. 2007, in Protostars and Planets V, ed. B. Reipurth, D. Jewitt, \& K. Keil, 573

Mischna, M. A., Kasting, J. F., Pavlov, A., \& Freedman, R. 2000, Icarus, 145, 546

Montes, D., López-Santiago, J., Gálvez, M. C., et al. 2001, MNRAS, 328, 45

O'Neill, C., Jellinek, A. M., \& Lenardic, A. 2007, Earth and Planetary Science Lett., 261, 20 
Paillet, J. 2006, Ph.D. Thesis, Université Paris XI

Pavlov, A. A., Kasting, J. F., Brown, L. L., Rages, K. A., \& Freedman, R. 2000, J. Geophys. Res., 105, 11981

Peale, S. J. 1999, ARA\&A, 37, 533

Pepin, R. O. 1991, Icarus, 92, 2

Pizzolato, N., Maggio, A., Micela, G., Sciortino, S., \& Ventura, P. 2003, A\&A 397, 147

Pollack, J. B., Kasting, J. F., Richardson, S. M., \& Poliakoff, K. 1987, Icarus, 71, 203

Rafikov, R. R. 2006, ApJ, 648, 666

Raymond, S. N., Quinn, T., \& Lunine, J. I. 2007, Astrobiology, 7, 66

Rivera, E. J., Lissauer, J. J., Butler, R. P., et al. 2005, ApJ, 634, 625

Rosing, M. T. 2005, Int. J. Astrobiol., 4, 9

Sagan, C., \& Chyba, C. 1997, Science, 276, 1217

Scalo, J., Kaltenegger, L., Segura, A. G., et al. 2007, Astrobiology, 7, 85

Schmitt, J. H. M. M., Fleming, T. A., \& Giampapa, M. S. 1995, ApJ, 450, 392

Schrag, D. P., Berner, R. A., Hoffman, P. F., \& Halverson, G. P. 2002 Geochemistry, Geophysics, Geosystems, 1

Segura, A., Kasting, J. F., Meadows, V., et al. 2005, Astrobiology, 5, 706

Selsis, F. 2004, in Extrasolar Planets: Today and Tomorrow, ed. J. Beaulieu, A. Lecavelier des Etangs, \& C. Terquem, ASP Conf. Ser., 321, 170
Selsis, F., Chazelas, B., Borde, P., et al. 2007, Icarus, 191, 453

Sleep, N. H., \& Zahnle, K. 2001, J. Geophys. Res., 106, 1373

Solomon, S. C., \& Head, J. W. 1991, Science, 252, 252

Sotin, C., Grasset, O., \& Moquet, A. 2007, Icarus, in press

Stauffer, J. R., Caillault, J.-P., Gagne, M., Prosser, C. F., \& Hartmann, L. W. 1994, ApJS, 91, 625

Stelzer, B., \& Neuhäuser, R. 2001, A\&A, 377, 538

Udry, S., Bonfils, X., Delfosse, X., et al. 2007, A\&A, 469, L43

Valencia, D., O'Connell, R. J., \& Sasselov, D. D. 2007a, ArXiv e-prints, 710

Valencia, D., Sasselov, D. D., \& O'Connell, R. J. 2007b, ApJ, 665, 1413

Vilhu, O., \& Walter, F. M. 1987, ApJ, 321, 958

Voges, W., Aschenbach, B., Boller, T., et al. 2000, IAU Circ., 7432, 1

Volonte, S., Laurance, R., Whitcomb, G., et al. 2000, Darwin: the Infrared space interferometer, Tech. Rep. (ESA)

von Bloh, W., Bounama, C., Cuntz, M., \& Franck, S. 2007, A\&A, 476, 1365

Walker, J. C. G., Hays, P. B., \& Kasting, J. F. 1981, J. Geophys. Res., 86, 9776

Williams, D. M., \& Pollard, D. 2002, Int. J. Astrobiol., 1, 61

Wood, B. E., Müller, H.-R., Zank, G. P., Linsky, J. L., \& Redfield, S. 2005, ApJ, 628, L143

Zahnle, K., Arndt, N., Cockell, C., et al. 2007, Space Sci. Rev., 127 